\title{
Crown ethers in the OPV series
}

\author{
Dirk Schwöppe, Bernhard Beile, and Herbert Meier* \\ Institute of Organic Chemistry \\ University of Mainz, Duesbergweg 10-14, 55099 Mainz, Germany \\ E-mail: hmeier@uni-mainz.de
}

\begin{abstract}
The present paper deals with the synthesis of oligo(1,4-phenylenevinylene)s (trimers, tetramers and a nonamer), which contain 1-3 (E)-stilbene crowns. A classical reaction route led first to stilbene crown subunits. Wittig-Horner reactions of diphosphonates with mono- or dialdehydes or of dialdehydes with mono- or diphosphonates yielded then the desired all-trans configured OPVs, which were designed for the complexation of metal cations.
\end{abstract}

Keywords: Absorption, condensation reactions, conjugated oligomers, crown ethers, WittigHorner reactions

\section{Introduction}

Among the conjugated oligomers, 1,4-phenylenevinylenes, OPVs (1) are prominent examples with various applications in materials science, such as light emitting diodes (LEDs), semiconductive or photoconductive devices, nonlinear optics, conversion of sunlight, formation of liquid crystals, etc. The OPV chains (Figure 1) can bear special functionalities, electrophores, chromophores, fluorophores and they can represent building blocks in star-shaped or dendritic compounds. See for example: Selected recently published work on OPV chains ${ }^{1-10}$ and reviews or monographs on this topic. ${ }^{11-25}$

Now we studied the preparation of OPVs $1(m=3,4,9)$, which contain crown ether segments of type $\mathbf{2}(n=1,3)$, which can be called stilbene crowns. To our best knowledge, until

now only the parent system $2(\mathrm{R}=\mathrm{H})$ and some simply substituted systems are known, ${ }^{26-32}$ but no higher conjugated stilbenoid crown $2(-\mathrm{R}:-\mathrm{CH}=\mathrm{CH}-)$ and particularly no OPV crown. 


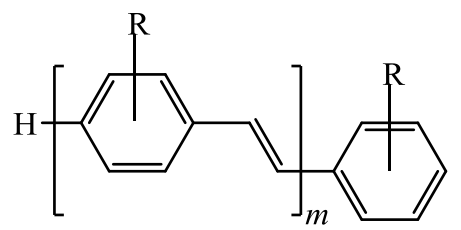

1

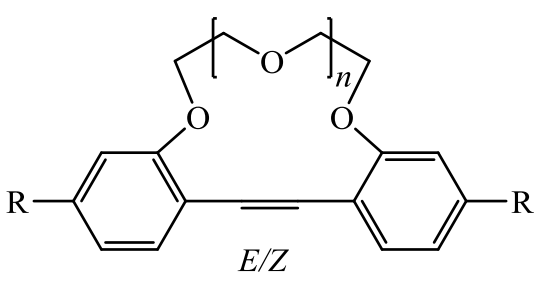

2

Figure 1. OPVs $1(m=2,3, \ldots)$ and stilbene crowns $2(n=1,2,3, \ldots)$.

OPVs, bearing stilbene crown units, are interesting in the context of sensor techniques, because their absorption and their fluorescence change by the complexation of metal cations. ${ }^{33}$ In comparison to stilbene, OPVs have some favourable properties, such as a bathochromically shifted, intense absorption and the corresponding fluorescence with an increased quantum yield. Moreover, OPVs do not show $E \rightarrow Z$ isomerization reactions on direct irradiation $\left(S_{0} \rightarrow S_{1}\right)$.

\section{Results and Discussion}

In order to get OPV crowns, we synthesized first the stilbene crowns $\mathbf{8 a}, \mathbf{b}$ and $\mathbf{9 a}, \mathbf{b}$ (Scheme 1), which bear substituents, that are suitable for the extension of the conjugated chain. The synthetic protocol for the parent stilbene crown was originally conceived by Gandour et al. ${ }^{26,27}$ and improved by Merz et al. ${ }^{28}$ We used a somewhat modified procedure: The methyl substituted salicylaldehyde $\mathbf{3}^{34}$ was first reacted with the twofold methylsulfonic acid ester $\mathbf{4 a}^{35,36}$ or $\mathbf{4} \mathbf{b}^{35,37}$ in the presence of sodium ethoxide to yield 5a,b. McMurry reactions led then to the tricyclic crowns $\mathbf{6 a}, \mathbf{b}$. The formation of the $\mathrm{CC}$ double bond showed a slight selectivity in favour of the $(E)$-configuration. The separation of the stereoisomers was performed for $\mathbf{6 a}$ by column chromatography and for $\mathbf{6 b}$ by complexation with $\mathrm{NaClO}_{4}$. (Z)-6b formed a more stable $\mathrm{Na}^{+}$ complex than $(E)-6 \mathbf{b}$ (see experimental part). However, it turned out that the $E / Z$-mixtures can be directly used for the next step, the Wohl-Ziegler bromination, in which a radical induced isomerization occured. The thermal equilibrium was then perfectly on the side of the $(E)$-isomers $\mathbf{7 a}, \mathbf{b}$, which were transformed by Arbusov reactions to the disphosphonates 8a,b. Oxidation of 7a,b with 2-nitropropane in the presence of sodium ethoxide yielded the dialdehydes 9a,b (Scheme 1). 


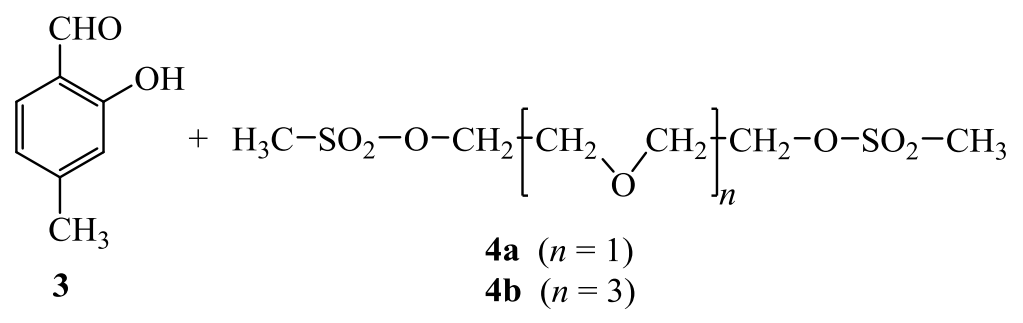<smiles>Cc1ccc(C=O)c(OCCOCCOc2cc(C)ccc2C=O)c1</smiles><smiles>CCOCCOc1cc(CBr)ccc1/C=C/c1ccc(CBr)cc1OCC</smiles>
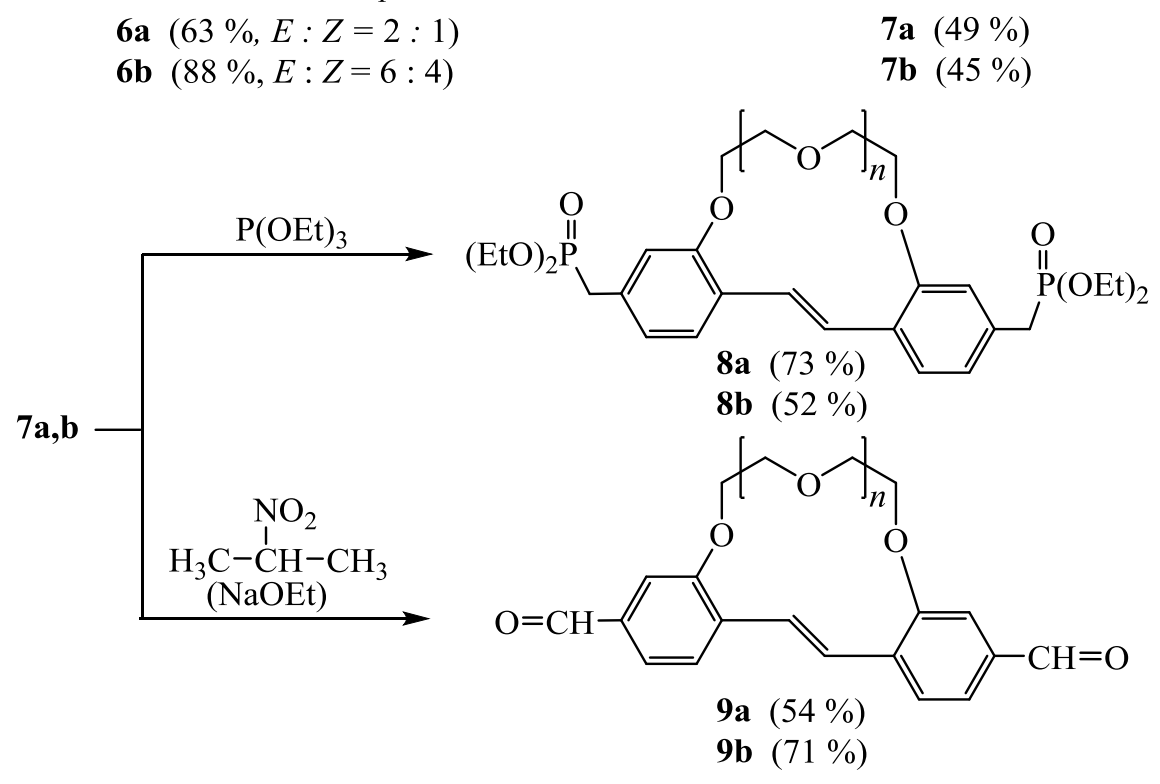

Scheme 1. Preparation of substituted stilbene crowns.

Twofold Wittig-Horner reactions of $\mathbf{8 a}, \mathbf{b}$ and the substituted benzaldehydes $\mathbf{1 0}^{38}, \mathbf{1 1}^{39}, \mathbf{1 2}^{40}$, $13^{41}, \mathbf{1 4}^{42}, \mathbf{1 5}^{40}, \mathbf{1 6}^{41}$ led to an extension of the conjugated chain in the all-trans configured $4,4^{\prime}$ distyrylstilbenes 17a,b-23a,b. Phase-transfer catalysis with Aliquat 336 in the presence of $\mathrm{KOH}$ gave yields between 48 and 66\% (Methode a), Scheme 2). 
a)

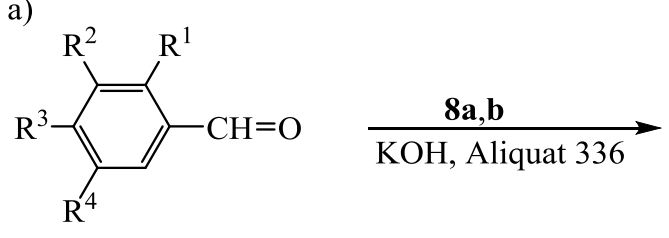

10-16

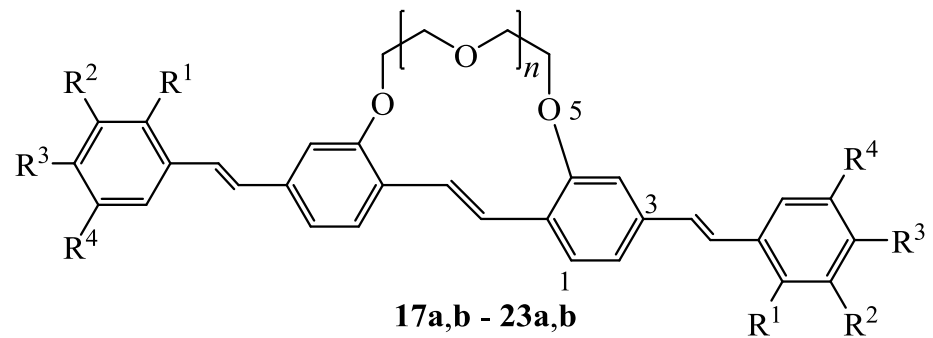

\begin{tabular}{|c|c|c|c|c|c|c|c|}
\hline & $\mathrm{R}^{1}$ & $\mathrm{R}^{2}$ & $\mathrm{R}^{3}$ & $\mathrm{R}^{4}$ & Product & $n$ & Yield \\
\hline 10 & $\mathrm{H}$ & $\mathrm{H}$ & $\mathrm{OCH}_{3}$ & $\mathrm{H}$ & $17 \mathbf{a}$ & 1 & 49 \\
\hline 10 & $\mathrm{H}$ & $\mathrm{H}$ & $\mathrm{OCH}_{3}$ & $\mathrm{H}$ & $17 b$ & 3 & 66 \\
\hline 11 & $\mathrm{H}$ & $\mathrm{OC}_{12} \mathrm{H}_{25}$ & $\mathrm{OC}_{12} \mathrm{H}_{25}$ & $\mathrm{OC}_{12} \mathrm{H}_{25}$ & $18 \mathbf{a}^{45}$ & 1 & 51 \\
\hline 11 & $\mathrm{H}$ & $\mathrm{OC}_{12} \mathrm{H}_{25}$ & $\mathrm{OC}_{12} \mathrm{H}_{25}$ & $\mathrm{OC}_{12} \mathrm{H}_{25}$ & $\mathbf{1 8 b}^{45}$ & 3 & 55 \\
\hline 12 & $\mathrm{OC}_{3} \mathrm{H}_{7}$ & $\mathrm{H}$ & $\mathrm{Br}$ & $\mathrm{OC}_{3} \mathrm{H}_{7}$ & $19 a$ & 1 & 64 \\
\hline 12 & $\mathrm{OC}_{3} \mathrm{H}_{7}$ & $\mathrm{H}$ & $\mathrm{Br}$ & $\mathrm{OC}_{3} \mathrm{H}_{7}$ & $19 b$ & 3 & 57 \\
\hline 13 & $\mathrm{OC}_{6} \mathrm{H}_{13}$ & $\mathrm{H}$ & $\mathrm{Br}$ & $\mathrm{OC}_{6} \mathrm{H}_{13}$ & $20 a$ & 1 & 49 \\
\hline 13 & $\mathrm{OC}_{6} \mathrm{H}_{13}$ & $\mathrm{H}$ & $\mathrm{Br}$ & $\mathrm{OC}_{6} \mathrm{H}_{13}$ & $20 \mathrm{~b}$ & 3 & 43 \\
\hline 14 & $\mathrm{H}$ & $\mathrm{H}$ & $\mathrm{CH}\left(\mathrm{OC}_{2} \mathrm{H}_{5}\right)_{2}$ & $\mathrm{H}$ & $21 \mathrm{a}$ & 1 & 56 \\
\hline 14 & $\mathrm{H}$ & $\mathrm{H}$ & $\mathrm{CH}\left(\mathrm{OC}_{2} \mathrm{H}_{5}\right)_{2}$ & $\mathrm{H}$ & $21 b$ & 3 & 62 \\
\hline 15 & $\mathrm{OC}_{3} \mathrm{H}_{7}$ & $\mathrm{H}$ & $\mathrm{CH}\left(\mathrm{OCH}_{3}\right)_{2}$ & $\mathrm{OC}_{3} \mathrm{H}_{7}$ & $22 a$ & 1 & 48 \\
\hline 15 & $\mathrm{OC}_{3} \mathrm{H}_{7}$ & $\mathrm{H}$ & $\mathrm{CH}\left(\mathrm{OCH}_{3}\right)_{2}$ & $\mathrm{OC}_{3} \mathrm{H}_{7}$ & $22 \mathrm{~b}$ & 3 & 48 \\
\hline 16 & $\mathrm{OC}_{6} \mathrm{H}_{13}$ & $\mathrm{H}$ & $\mathrm{CH}\left(\mathrm{OCH}_{3}\right)_{2}$ & $\mathrm{OC}_{6} \mathrm{H}_{13}$ & $23 a$ & 1 & 56 \\
\hline 16 & $\mathrm{OC}_{6} \mathrm{H}_{13}$ & $\mathrm{H}$ & $\mathrm{CH}\left(\mathrm{OCH}_{3}\right)_{2}$ & $\mathrm{OC}_{6} \mathrm{H}_{13}$ & $23 b$ & 3 & 63 \\
\hline
\end{tabular}

b)

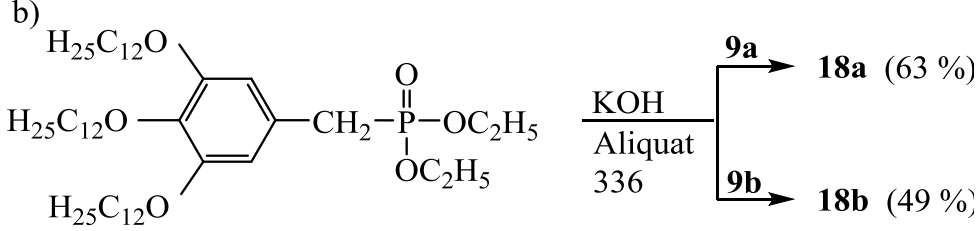

24

Scheme 2. Preparation of distyrylstilbene crowns.

The products 18a and 18b were also obtained by "inverse" Wittig-Horner processes of the dialdehydes 9a,b and the monophosphonate $\mathbf{2 4}^{43,44}$ (Methode b), Scheme 2). The OPV trimers 17a,b-23a,b have all absorption maxima in the visible region (see experimental part), whereas for example the $\lambda_{\max }$ values of $(E)-\mathbf{6 a}, \mathbf{b}$ and $(Z)-\mathbf{6 a}, \mathbf{b}$ are in the UV.

Acidic hydrolysis of the acetals 21a,b-23a,b yielded the dialdehydes 25a,b-27a,b (Scheme $3)$. 


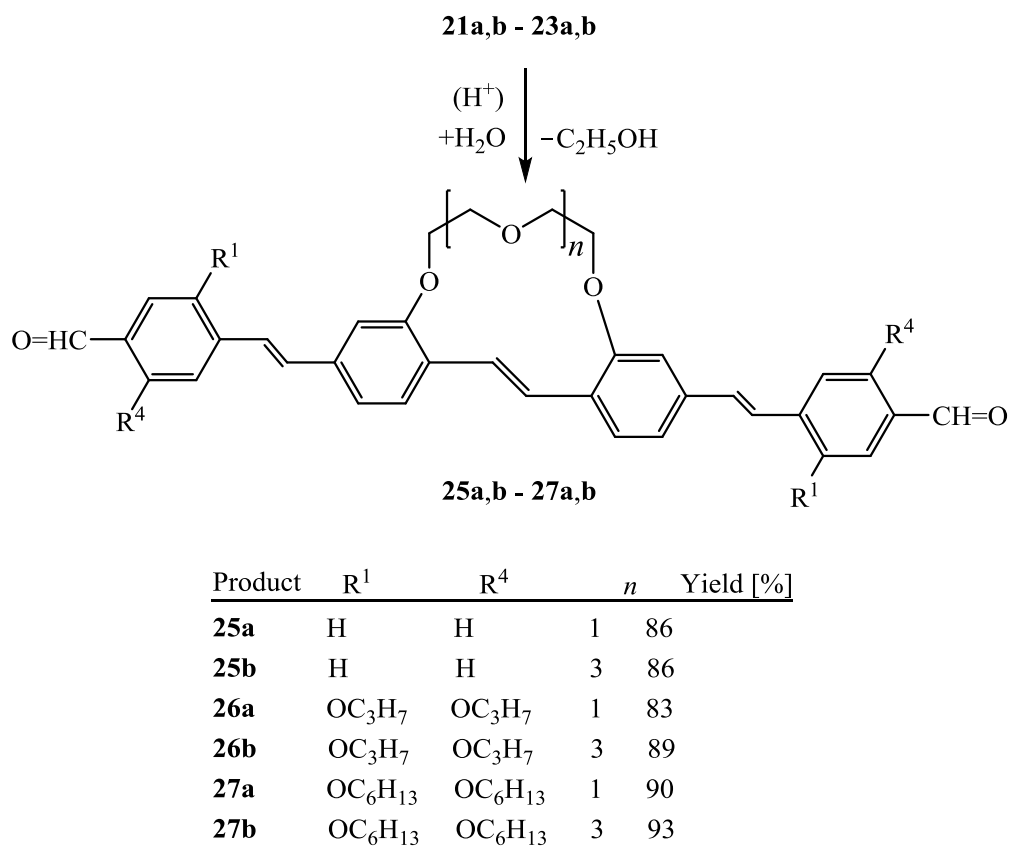

Scheme 3. Cleavage of acetals 21a,b-23a,b $\rightarrow 25 a, b-27 a, b$.

All stilbene crowns $\mathbf{6 a}, \mathbf{b}-\mathbf{9 a}, \mathbf{b}, \mathbf{1 7 a}, \mathbf{b}-\mathbf{2 3 a}, \mathbf{b}$ and $25 \mathbf{a}, \mathbf{b}-\mathbf{2 7} \mathbf{a}, \mathbf{b}$ with $(E)$-configurations in the macrocyclic ring show fast rotations of the single bonds adjacent to the olefinic double bond. Thus, inner and outer olefinic $\mathrm{H}$ atoms exchange and the $C_{\mathrm{s}}$ symmetry becomes a de facto $C_{2 \mathrm{v}}$ symmetry. The resonance of the olefinic protons in the $(E)$-configured 13-membered ring systems 6a-9a, 17a-23a and 25a-27a is at much lower field $(\delta>7.7 \mathrm{ppm})$ than in the $(E)$ configured 19-membered crowns $\mathbf{6 b}-\mathbf{9 b}, \mathbf{1 7} \mathbf{b}-\mathbf{2 3 b}$ and 25b-27b $(\delta<7.5 \mathrm{ppm})$. This cannot be a result of different planarity, since the resonance of the olefinic protons is at $\delta=7.49 \mathrm{ppm}$ in $(E)$ 2,2'-dimethoxy stilbene which is a planar model compound. ${ }^{46,47} \mathrm{We}$ assume that the down-field shift in the 13-membered rings is owing to transannular steric interactions in this constrained ring systems.

OPVs have the principal problem of a low solubility. Alkyl or better alkoxy substituents improve the solubility. Therefore, we prepared additionally the diphosphonates $\mathbf{2 8}^{48}$ and $\mathbf{2 9}^{49}$ and reacted them with an excess amount of $\mathbf{9 a}, \mathbf{b}$ (Scheme 4). The portion, which was soluble in $\mathrm{CH}_{2} \mathrm{Cl}_{2}$, consisted of the 1,4-phenylenevinylene tetramers 30a,b and 31a,b with formyl end groups. Phosphonate end groups could not be found. The insoluble major part consisted of higher OPVs. 


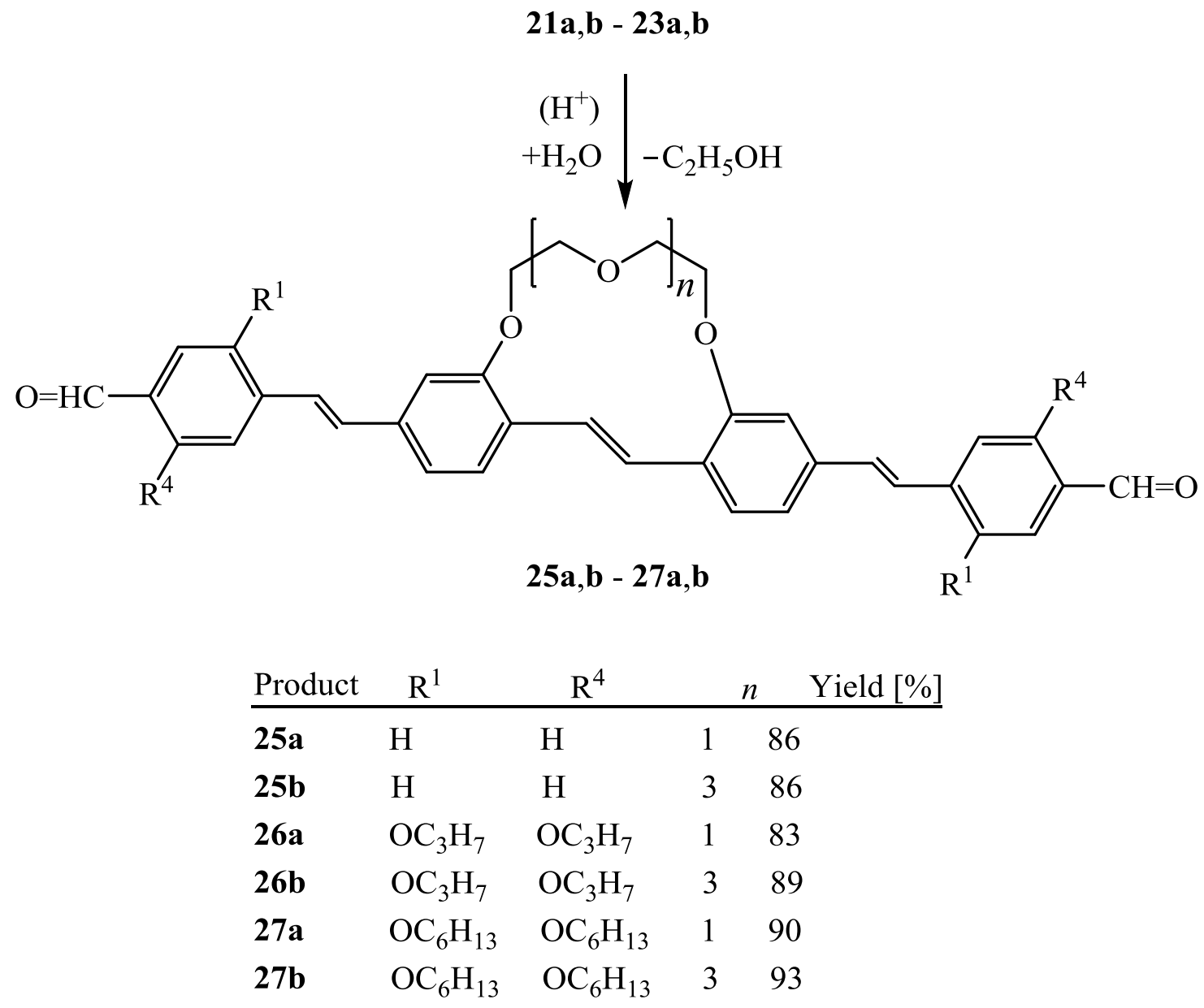

Scheme 4. Synthesis of double stilbene crowns connected by a 1,4-divinylenephenylene unit.

The tricyclic crowns in 30a,b and 31a,b show the same molecular dynamics as the stilbene crowns discussed above. However, the $C_{2 \mathrm{~h}}$ symmetry is not affected by this process. Each crown contains four $(n=1)$ or eight $(n=2)$ different $\mathrm{OCH}_{2}$ groups - documented for example by the number of ${ }^{13} \mathrm{C}$ NMR signals.

The compounds 8, 9, 19, 20 and 25-31 are bifunctional systems whose terminal functionalities can be used for condensation reactions: The dibromides $\mathbf{1 9}$ and $\mathbf{2 0}$ for Yamamoto or Heck coupling reactions, the dialdehydes $\mathbf{9 , 2 5 - 2 7 , 3 0}$ and $\mathbf{3 1}$ for iterative McMurry reactions or repeated Wittig-Horner reactions with the diphosphonates 8, 28 and 29. In the two latter cases 1,4-phenylenevinylenes of oligomer type $\mathbf{3 2}$ or $\mathbf{3 3}$ should result. Our first attempts in this direction led predominantly to insoluble and intractable materials, which we did not study further. The Wittig-Horner reaction of dialdehyde 27a and diphosphonate 8a was in so far an exception as a small portion of an oligomer could be obtained which was the only soluble component in hot $\mathrm{CHCl}_{3}$. It was essentially the 2:1 adduct of 27a and 8a and had the 1,4phenylenevinylene nonamer structure 34 (Scheme 5). The FD-MS technique showed a distinct molecular ion of $m / z=2160$ for $\mathrm{C}_{140} \mathrm{H}_{174} \mathrm{O}_{19}$. 
All monodisperse OPVs bearing crown ether units were characterized by their ${ }^{1} \mathrm{H}$ and ${ }^{13} \mathrm{C}$ NMR data and by MS spectra. Their electronic excitation resembles that of alkoxy substituted oligo(1,4-phenylenevinylene)s. ${ }^{16,50}$ The trimers $\mathbf{1 7 - 2 3}$ and 25-27 have in $\mathrm{CH}_{2} \mathrm{Cl}_{2} \lambda_{\max }$ values between 399 and $438 \mathrm{~nm}$, the tetramers $\mathbf{3 0}$ and $\mathbf{3 1}$ between 443 and $447 \mathrm{~nm}$ and the nonamer 34 has its absorption maximum at $457 \mathrm{~nm}$. The change of the absorption and fluorescence spectra by complexation with alkali cations ( $\mathrm{Li}, \mathrm{Na}, \mathrm{K}, \mathrm{Rb}, \mathrm{Cs}$ ) is under investigation.

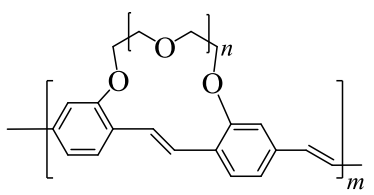

$32(n=1,3)$

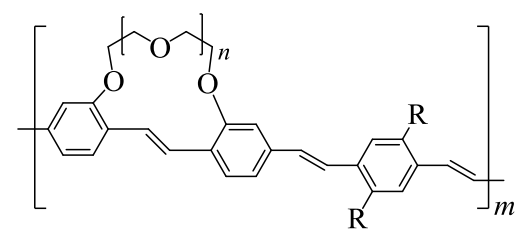

$33(n=1,3$;

$\left.\left.\mathrm{R}=\mathrm{OC}_{3} \mathrm{H}_{7}\right), \mathrm{R}=\mathrm{OC}_{6} \mathrm{H}_{13}\right)$

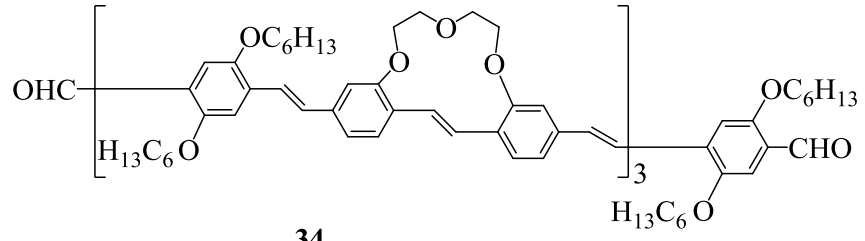

34

Scheme 5. Higher OPVs containing (E)-stilbene crown ether structures.

\section{Conclusions}

In conclusion, the preparation of special oligo(1,4-phenylenevinylene)s is described here. On the basis of Wittig-Horner reactions, the trimers 17-23 and 25-27, the tetramers 30, 31 and a nonamer 34 were obtained. The conjugated chains contain one, two or three $(E)$-stilbene crown ether subunits, which were designed for the complexation of alkali cations. 


\section{Experimental Section}

General. UV/Vis: Perkin-Elmer Lambda 15. ${ }^{1} \mathrm{H}$ NMR and ${ }^{13} \mathrm{C}$ NMR: Bruker AM 400, $\mathrm{CDCl}_{3}$ as solvent if not otherwise stated, TMS as internal standard. EI- and FD-MS: Varian MAT CH7A. The melting points were measured on a Büchi apparatus and are not corrected.

2,2' -[Oxybis(2,1-ethanediyloxy)]bis(4-methylbenzaldehyde) (5a). Aldehyde $\mathbf{3}^{27}$ (50.0 g, 0.37 $\mathrm{mol}$ ), dissolved in $100 \mathrm{~mL}$ of ethanol, was slowly added to a solution of sodium ethoxide, obtained from sodium $(10.35 \mathrm{~g}, 0.45 \mathrm{~mol})$ and $300 \mathrm{~mL}$ of ethanol. After $12 \mathrm{~h}$ stirring at room temperature, the formed crystals were filtered off and washed several times with cold ethanol. The dried sodium phenolate was then added to the dimethylsulfonate $4 \mathbf{a}^{28,29}$ (41.97 $\mathrm{g}, 0.16 \mathrm{~mol}$ ) in $500 \mathrm{~mL}$ of dry toluene. After $48 \mathrm{~h}$ of heating to reflux, the formed precipitate was filtered off and washed several times with toluene and then with $2 \%$ aqueous $\mathrm{NaOH}$. The dried product 5a was recrystallized from diethylether. Yield: $40.4 \mathrm{~g}(72 \%), \mathrm{mp} 79{ }^{\circ} \mathrm{C} .{ }^{1} \mathrm{H} \mathrm{NMR}\left(\mathrm{CDCl}_{3}\right): \delta 2.34$ (s, 6H, $\left.\mathrm{CH}_{3}\right), 3.90-4.00\left(\mathrm{~m}, 4 \mathrm{H}, \mathrm{OCH}_{2}\right), 4.16-4.26\left(\mathrm{~m}, 4 \mathrm{H}, \mathrm{OCH}_{2}\right), 6.75(\mathrm{~s}, 2 \mathrm{H}, \mathrm{H}-3), 6.79\left(\mathrm{~d},{ }^{3} J\right.$ $7.8 \mathrm{~Hz}, 2 \mathrm{H}, \mathrm{H}-5), 7.67$ (d, $\left.{ }^{3} \mathrm{~J} 7.8 \mathrm{~Hz}, 2 \mathrm{H}, \mathrm{H}-6\right), 10.39$ (s, 2H, CHO). ${ }^{13} \mathrm{C} \mathrm{NMR}\left(\mathrm{CDCl}_{3}\right): \delta 22.3$ $\left(\mathrm{CH}_{3}\right)$, 68.3, $69.9\left(\mathrm{OCH}_{2}\right), 113.4(\mathrm{C}-3), 122.1$ (C-5), 122.9 (C-1), $128.4(\mathrm{C}-6), 147.4(\mathrm{C}-4), 161.2$ (C-2), 189.3 (CHO). MS (FD), $m / z(\%) 342$ (100) $[\mathrm{M}]^{+}$. Anal. Calcd for $\mathrm{C}_{20} \mathrm{H}_{22} \mathrm{O}_{5}$ (342.4): C, 70.16; H, 6.41. Found: C, 70.06; H, 6.51.

2,2'-[Oxybis(2,1-ethanediyloxy-2,1-ethanediyloxy)]bis(4-methylbenzaldehyde) (5b). The preparation was performed according to 5a. $45.4 \mathrm{~g}(0.29 \mathrm{~mol})$ of the sodium phenolate and 34.2 $\mathrm{g}(0.13 \mathrm{~mol})$ of the dimethyl sulfonate $4 \mathrm{~b}$ yielded after $48 \mathrm{~h}$ heating to reflux in $500 \mathrm{~mL}$ of toluene $25.6 \mathrm{~g}(58 \%)$ of $\mathbf{5 b}$ as a viscous oil. ${ }^{1} \mathrm{H} \mathrm{NMR}\left(\mathrm{CDCl}_{3}\right): \delta 2.32\left(\mathrm{~s}, 6 \mathrm{H}, \mathrm{CH}_{3}\right), 3.60-3.70$ $\left(\mathrm{m}, 8 \mathrm{H}, \mathrm{OCH}_{2}\right), 3.79-3.89\left(\mathrm{~m}, 4 \mathrm{H}, \mathrm{OCH}_{2}\right), 4.11-4.21\left(\mathrm{~m}, 4 \mathrm{H}, \mathrm{OCH}_{2}\right), 6.72(\mathrm{~s}, 2 \mathrm{H}, \mathrm{H}-3), 6.76(\mathrm{~d}$, $\left.{ }^{3} J 7.8 \mathrm{~Hz}, 2 \mathrm{H}, \mathrm{H}-5\right), 7.65$ (d, $\left.{ }^{3} J 7.8 \mathrm{~Hz}, 2 \mathrm{H}, \mathrm{H}-6\right), 10.38$ (s, 2H, CHO). ${ }^{13} \mathrm{C} \mathrm{NMR}\left(\mathrm{CDCl}_{3}\right): \delta$ $22.3\left(\mathrm{CH}_{3}\right), 68.1,69.5,70.7,71.0\left(\mathrm{OCH}_{2}\right), 113.4(\mathrm{C}-3), 122.0(\mathrm{C}-5), 122.9(\mathrm{C}-1), 128.2(\mathrm{C}-6)$, 147.3 (C-4), 161.3 (C-2), 189.4 (CHO). MS (FD), $m / z(\%) 430$ (100) $[\mathrm{M}]^{+}$. Anal. Calcd for $\mathrm{C}_{24} \mathrm{H}_{30} \mathrm{O}_{7}$ (430.5): C, 66.96; H, 7.02. Found: C, 66.60; H, 6.83.

3,13-Dimethyl-6,7,9,10-tetrahydro-5,8,11-trioxa-dibenzo[a,e $]$ cyclotridecene (6a). A reaction vessel with a high dilution equipment was filled under Ar with $600 \mathrm{~mL}$ of dry THF. At $0{ }^{\circ} \mathrm{C} 89.6$ $\mathrm{g}(0.47 \mathrm{~mol}) \mathrm{TiCl}_{4}$ were added dropwise before a $\mathrm{Zn} / \mathrm{Cu}$ couple $(105.4 \mathrm{~g}, 0.94 \mathrm{~mol})$ was added. The originally light yellow solution turned dark. After $30 \mathrm{~min}$ boiling ( $110{ }^{\circ} \mathrm{C}$ bath temperature), dialdehyde 5a (40.4 g, $0.12 \mathrm{~mol}$ ), dissolved in $300 \mathrm{~mL}$ of dry THF, was dropped in $30 \mathrm{~h}$ through a high-precision funnel into the reflux of the solvent. The mixture was boiled for another hour before $800 \mathrm{~mL}$ of an aqueous $10 \% \mathrm{Na}_{2} \mathrm{CO}_{3}$ solution and $800 \mathrm{~mL}$ of diethylether were added. Filtration over Celit gave an organic phase which was dried over $\mathrm{MgSO}_{4}$ and evaporated. The solid residue $(23.15 \mathrm{~g}, 63 \%)$ consisted of a 2:1 mixture of $(E)-$ and $(Z)-6 a . M S(E I)(70 \mathrm{ev}), \mathrm{m} / \mathrm{z}$ 
(\%) 310 (100) $[\mathrm{M}]^{+}$. Anal. Calcd for $\mathrm{C}_{20} \mathrm{H}_{22} \mathrm{O}_{3}$ (310.4): C, 77.39; H, 7.14. Found: C, 77.31; H, 7.18 .

The separation of the stereoisomers was possible by column chromatography $\left(\mathrm{SiO}_{2}, \mathrm{CH}_{2} \mathrm{Cl}_{2}\right)$, but the next step could be made with the $E / Z$ mixture as well.

(E)-6a: Colorless powder, mp $136{ }^{\circ} \mathrm{C} .{ }^{1} \mathrm{H}$ NMR $\left(\mathrm{CDCl}_{3}\right): \delta 2.32\left(\mathrm{~s}, 6 \mathrm{H}, \mathrm{CH}_{3}\right), 3.85-3.94(\mathrm{~m}$, $\left.4 \mathrm{H}, \mathrm{OCH}_{2}\right), 4.09-4.19\left(\mathrm{~m}, 4 \mathrm{H}, \mathrm{OCH}_{2}\right), 6.80$ (s, 2H, H-4, H-12), 6.83 (d, ${ }^{3} J 7.9 \mathrm{~Hz}, 2 \mathrm{H}, \mathrm{H}-2, \mathrm{H}-$ 14), 7.36 (d, $\left.{ }^{3} J \quad 7.9 \mathrm{~Hz}, 2 \mathrm{H}, \mathrm{H}-1, \mathrm{H}-15\right), 7.73$ (s, 2H, H-16, H-17). ${ }^{13} \mathrm{C} \mathrm{NMR}\left(\mathrm{CDCl}_{3}\right): \delta 21.3$ $\left(\mathrm{CH}_{3}\right), 70.1,70.7\left(\mathrm{OCH}_{2}\right), 118.1,123.4,125.4,127.6$ (aromat. and olefin. $\left.\mathrm{CH}\right), 126.6$, 137.9, 157.0 (aromat. $\left.\mathrm{C}_{\mathrm{q}}\right)$. UV $\left(\mathrm{CH}_{2} \mathrm{Cl}_{2}\right): \lambda_{\max }(\varepsilon) 328 \mathrm{~nm}\left(20100 \mathrm{M}^{-1} \mathrm{~cm}^{-1}\right)$.

(Z)-6a: Oil. ${ }^{1} \mathrm{H}$ NMR $\left(\mathrm{CDCl}_{3}\right): \delta 2.25\left(\mathrm{~s}, 6 \mathrm{H}, \mathrm{CH}_{3}\right), 3.67-3.77\left(\mathrm{~m}, 4 \mathrm{H}, \mathrm{OCH}_{2}\right), 3.94-4.03(\mathrm{~m}, 4 \mathrm{H}$, $\mathrm{OCH}_{2}$ ), 6.61-6.63 (m, 6H, H-2, H-4, H-12, H-14, H-16, H-17), 6.88 (d, ${ }^{3} J 7.6$ Hz, 2H, H-1, H15). ${ }^{13} \mathrm{C} \mathrm{NMR}\left(\mathrm{CDCl}_{3}\right): \delta 20.9\left(\mathrm{CH}_{3}\right), 69.7,71.0\left(\mathrm{OCH}_{2}\right), 116.3,122.2,127.2,130.1$ (aromat. and olefin. $\mathrm{CH}), 126.6,137.2,155.8$ (aromat. $\left.\mathrm{C}_{\mathrm{q}}\right) \cdot \mathrm{UV}\left(\mathrm{CH}_{2} \mathrm{Cl}_{2}\right): \lambda_{\max }(\varepsilon) 290 \mathrm{~nm}\left(6300 \mathrm{M}^{-1} \mathrm{~cm}^{-1}\right)$.

3,19-Dimethyl-6,7,9,10,12,13,15,16-octahydro-5,8,11,14,17-pentaoxa-dibenzo[a,e]cyclononadecene (6b). Preparation according to 6a. The oily product $(39.9 \mathrm{~g}, 88 \%)$ obtained from $89.5 \mathrm{~g}$ $(0.46 \mathrm{~mol}) \mathbf{5 b}$ was obtained as a 6:4 mixture of $(E)$ - and $(Z)-6 \mathbf{b}$ after a reaction time of $3 \mathrm{~d}$. MS (FD), $m / z(\%) 398$ (100) [M] ${ }^{+}$. Anal. Calcd for $\mathrm{C}_{24} \mathrm{H}_{30} \mathrm{O}_{5}$ (398.5): C, 72.34; H, 7.59. Found: C, 71.97; H, 7.39.

The product could be used directly for the next step. The separation of the stereoisomers was performed with the help of the complex formation with $\mathrm{NaClO}_{4}$. The oil, dissolved in $300 \mathrm{~mL}$ of dry THF, was treated with $\mathrm{NaClO}_{4}(13.0 \mathrm{~g}, 0.11 \mathrm{~mol})$ in $200 \mathrm{~mL}$ of THF. After stirring at room temperature for $6 \mathrm{~h}$, the formed precipitate was filtered off and dissolved in $300 \mathrm{~mL}$ of water. The aqueous solution was then continuously extracted with $\mathrm{CH}_{2} \mathrm{Cl}_{2}$. The organic phase was dried $\left(\mathrm{Na}_{2} \mathrm{SO}_{4}\right)$ and the solvent evaporated. The residue consisted of $15.6 \mathrm{~g}(34 \%)$ of pure $(Z)$-isomer which formed the more stable $\mathrm{Na}^{+}$complex.

(Z)-6b: Viscous oil. ${ }^{1} \mathrm{H}$ NMR $\left(\mathrm{CDCl}_{3}\right): \delta 2.28\left(\mathrm{~s}, 6 \mathrm{H}, \mathrm{CH}_{3}\right), 3.56-3.76\left(\mathrm{~m}, 12 \mathrm{H}, \mathrm{OCH}_{2}\right), 3.94-$ 4.04 (m, 4H, $\mathrm{OCH}_{2}$ ), 6.60 (d, $\left.{ }^{3} J 7.8 \mathrm{~Hz}, 2 \mathrm{H}, \mathrm{H}-2\right), 6.65$ (s, 2H, H-4, H-18), 6.71 (s, 2H, H-22, H23), 7.09 (d, $\left.{ }^{3} J 7.8 \mathrm{~Hz}, 2 \mathrm{H}, \mathrm{H}-1, \mathrm{H}-21\right) .{ }^{13} \mathrm{C} \mathrm{NMR}\left(\mathrm{CDCl}_{3}\right): \delta 21.5\left(\mathrm{CH}_{3}\right), 69.0,69.5,70.7,71.1$ $\left(\mathrm{OCH}_{2}\right), 113.9,121.3,124.8,129.1$ (aromat. and olefin. $\mathrm{CH}$ ), 125.3, 138.0, 155.8 (aromat. $\mathrm{C}_{\mathrm{q}}$ ). $\mathrm{UV}\left(\mathrm{CH}_{2} \mathrm{Cl}_{2}\right): \lambda_{\max }(\varepsilon) 302 \mathrm{~nm}\left(7400 \mathrm{M}^{-1} \mathrm{~cm}^{-1}\right)$.

The filtered THF solution was evaporated and the residue dissolved in $300 \mathrm{~mL}$ of $\mathrm{CH}_{2} \mathrm{Cl}_{2}$. The solution was washed twice with the same amount of $\mathrm{H}_{2} \mathrm{O}$ and the dried organic phase $\left(\mathrm{Na}_{2} \mathrm{SO}_{4}\right)$ evaporated. The remaining solid was recrystallized from diethylether to which at the boiling point petroleum ether (bp $40-70{ }^{\circ} \mathrm{C}$ ) was added, till the precipitation started.

(E)-6b (24.3 g, 54\%) was obtained as a colorless solid, mp $121{ }^{\circ} \mathrm{C} .{ }^{1} \mathrm{H}$ NMR $\left(\mathrm{CDCl}_{3}\right): \delta 2.32(\mathrm{~s}$, $\left.6 \mathrm{H}, \mathrm{CH}_{3}\right), 3.63-3.79\left(\mathrm{~m}, 8 \mathrm{H}, \mathrm{OCH}_{2}\right), 3.94-4.04\left(\mathrm{~m}, 4 \mathrm{H}, \mathrm{OCH}_{2}\right), 4.11-4.21\left(\mathrm{~m}, 4 \mathrm{H}, \mathrm{OCH}_{2}\right), 6.70$ (s, 2H, H-4, H-18), 6.76 (d, ${ }^{3} J 7.8$ Hz, 2H, H-2, H-20), 7.31 (d, ${ }^{3} J$ 7.8 Hz, 2H, H-1, H-21), 7.35 (s, 2H, H-22, H-23). ${ }^{13} \mathrm{C} \mathrm{NMR}\left(\mathrm{CDCl}_{3}\right): \delta 21.6\left(\mathrm{CH}_{3}\right), 67.7,69.9,71.2,71.5\left(\mathrm{OCH}_{2}\right), 112.9$, 
121.5, 128.6, 126.4 (aromat. and olefin. $\mathrm{CH}$ ), 125.0, 138.0, 156.4 (aromat. $\mathrm{C}_{\mathrm{q}}$ ). $\mathrm{UV}\left(\mathrm{CH}_{2} \mathrm{Cl}_{2}\right.$ ): $\lambda_{\max }(\varepsilon) 328 \mathrm{~nm}\left(9800 \mathrm{M}^{-1} \mathrm{~cm}^{-1}\right)$.

(E)-3,13-Bisbromomethyl-6,7,9,10-tetrahydro-5,8,11-trioxa-dibenzo[a,e $]$ cyclotridecene (7a). $N$-Bromosuccinimide $(11.5 \mathrm{~g}, 64.4 \mathrm{mmol}), 6 \mathrm{a}(10.0 \mathrm{~g}, 32.2 \mathrm{mmol})$ and azobisisobutyronitrile (AIBN) $(1.00 \mathrm{~g}, 6.0 \mathrm{mmol})$ were heated in $100 \mathrm{~mL}$ of dry $\mathrm{CCl}_{4}$ to reflux. After $2 \mathrm{~h}$ the formed succinimide was filtered off and the solvent evaporated. The residue was recrystallized from $\mathrm{CHCl}_{3}$. Yellow crystals. Yield: $7.38 \mathrm{~g}(49 \%)$, mp $186{ }^{\circ} \mathrm{C} .{ }^{1} \mathrm{H}$ NMR $\left(\mathrm{CDCl}_{3}\right): \delta$ 3.85-3.94 (m, $\left.4 \mathrm{H}, \mathrm{OCH}_{2}\right), 4.10-4.20$ (m, 4H, $\mathrm{OCH}_{2}$ ), 4.45 (s, 4H, $\mathrm{CH}_{2} \mathrm{Br}$ ), 7.00 (s, 2H, H-4, H-12), 7.03 (d, ${ }^{3} J$ $7.9 \mathrm{~Hz}, 2 \mathrm{H}, \mathrm{H}-2, \mathrm{H}-14), 7.42$ (d, $\left.{ }^{3} \mathrm{~J} 7.9 \mathrm{~Hz}, 2 \mathrm{H}, \mathrm{H}-1, \mathrm{H}-15\right), 7.71$ (s, 2H, H-16, H-17). ${ }^{13} \mathrm{C}$ NMR $\left(\mathrm{CDCl}_{3}\right): \delta 33.6\left(\mathrm{CH}_{2} \mathrm{Br}\right), 70.1,70.9\left(\mathrm{OCH}_{2}\right), 118.0,123.4,126.6,128.3$ (aromat. and olefin. $\mathrm{CH}), 129.5,137.7,157.3$ (aromat. $\mathrm{C}_{\mathrm{q}}$ ). MS (FD), $\mathrm{m} / z(\%)$ 466, 468, $470(100)[\mathrm{M}]^{+}, \mathrm{Br}_{2}$ pattern. Anal. Calcd for $\mathrm{C}_{20} \mathrm{H}_{20} \mathrm{Br}_{2} \mathrm{O}_{3}$ (468.2): C, 51.31; H, 4.31; Br, 34.31. Found: C, 51.39; H, 4.21; $\mathrm{Br}$, 33.97.

(E)-3,19-Bisbromomethyl-6,7,9,10,12,13,15,16-octahydro-5,8,11,14,17-dibenzo[a,e $]$ cyclononadecene (7b). Preparation according to 7a. The heating was extended to $5 \mathrm{~h}$. Recrystallization from diethylether/ $\mathrm{CHCl}_{3} 2: 1$ gave a yellow solid, which melted at $145{ }^{\circ} \mathrm{C}$. Yield: $2.61 \mathrm{~g}(45 \%)$ from $4.18 \mathrm{~g}(10.5 \mathrm{mmol}) 6 \mathrm{6} .{ }^{1} \mathrm{H}$ NMR $\left(\mathrm{CDCl}_{3}\right): \delta \quad 3.65-3.75(\mathrm{~m}, 8 \mathrm{H}$, $\left.\mathrm{OCH}_{2}\right), 3.93-4.03\left(\mathrm{~m}, 4 \mathrm{H}, \mathrm{OCH}_{2}\right), 4.15-4.25\left(\mathrm{~m}, 4 \mathrm{H}, \mathrm{OCH}_{2}\right), 4.47$ (s, $\left.4 \mathrm{H}, \mathrm{CH}_{2} \mathrm{Br}\right), 6.90(\mathrm{~s}, 2 \mathrm{H}$, H-4, H-18), 6.95 (d, $\left.{ }^{3} J 7.7 \mathrm{~Hz}, 2 \mathrm{H}, \mathrm{H}-2, \mathrm{H}-20\right), 7.35$ (d, $\left.{ }^{3} J 7.7 \mathrm{~Hz}, 2 \mathrm{H}, \mathrm{H}-1, \mathrm{H}-21\right), 7.37$ (s, 2H, $\mathrm{H}-22, \mathrm{H}-23) .{ }^{13} \mathrm{C}$ NMR $\left(\mathrm{CDCl}_{3}\right): \delta 33.9\left(\mathrm{CH}_{2} \mathrm{Br}\right), 67.9,69.8,71.3,71.5\left(\mathrm{OCH}_{2}\right), 112.7,121.6$, 127.5, 129.2 (aromat. and olefin. $\mathrm{CH}$ ), 127.9, 137.8, 156.7 (aromat. $\mathrm{C}_{\mathrm{q}}$ ). MS (FD), $\mathrm{m} / \mathrm{z}$ (\%) 554, 556, 558 (100) $[\mathrm{M}]^{+} \cdot \mathrm{Br}_{2}$ pattern. Anal. Calcd for $\mathrm{C}_{24} \mathrm{H}_{28} \mathrm{Br}_{2} \mathrm{O}_{5}$ (556.3): C, 51.82; H. 5.07; Br, 28.73. Found: C, 51.44; H, 4.92; Br, 29.13.

[(E)-13-(Diethoxy-phosphorylmethyl)-6,7,9,10-tetrahydro-5,8,11-trioxa-dibenzo[a,e]cyclotridecene-3-ylmethyl]-phosphonic acid diethyl ester (8a). Dibromide 7a (3.0 g, $6.4 \mathrm{mmol})$ and triethylphosphite $(2.919 \mathrm{~g}, 16.0 \mathrm{mmol})$ were heated to $160{ }^{\circ} \mathrm{C}$, so that the formed bromoethane was evaporated. After $6 \mathrm{~h}$ the excess triethylphosphite was distilled off at $1.0 \mathrm{kPa}$. The residue was purified by column chromatography $(4 \times 40 \mathrm{~cm} \mathrm{SiO}$, ethyl acetate/methanol 10:1). Yield: $2.70 \mathrm{~g}(73 \%)$. Colorless solid, mp $88{ }^{\circ} \mathrm{C} .{ }^{1} \mathrm{H}$ NMR $\left(\mathrm{CDCl}_{3}\right): \delta 1.24\left(\mathrm{t},{ }^{3} \mathrm{~J} 7.3 \mathrm{~Hz}, 12 \mathrm{H}, \mathrm{CH}_{3}\right)$, $3.09\left(\mathrm{~d},{ }^{2} \mathrm{~J}(\mathrm{P}, \mathrm{H}) 21.5 \mathrm{~Hz}, 4 \mathrm{H}, \mathrm{CH}_{2} \mathrm{P}\right), 3.87-4.04\left(\mathrm{~m}, 12 \mathrm{H}, \mathrm{POCH}_{2}\right.$ and $\left.\mathrm{OCH}_{2}\right), 4.06-4.17(\mathrm{~m}, 4 \mathrm{H}$, $\mathrm{OCH}_{2}$ ), 6.85-6.96 (m, 4H, H-2, H-4, H-12, H-14), 7.38 (d, ${ }^{3} J$ 8.1 Hz, 2H, H-1, H-15), 7.74 (s, 2H, H-16, H-17). ${ }^{13} \mathrm{C}$ NMR $\left(\mathrm{CDCl}_{3}\right): \delta 16.3\left(\mathrm{CH}_{3}\right), 33.7\left(\mathrm{~d},{ }^{1} J(\mathrm{P}, \mathrm{C}) \quad 137.2 \mathrm{~Hz}, \mathrm{CH}_{2} \mathrm{P}\right), 62.2$ $\left(\mathrm{POCH}_{2}\right), 70.2,70.8\left(\mathrm{OCH}_{2}\right), 118.7,124.1,126.0,127.9$ (aromat. and olefin. $\mathrm{CH}$ ), 128.0, 131.5, 157.2 (aromat. $\mathrm{C}_{\mathrm{q}}$ ). MS (FD), $m / z(\%) 582(100)[\mathrm{M}]^{+}$. Anal. Calcd for $\mathrm{C}_{28} \mathrm{H}_{40} \mathrm{O}_{9} \mathrm{P}_{2}$ (582.6): $\mathrm{C}$, 57.73; H, 6.92. Found: C, 57.63; H, 7.15. 
[(E)-19-(Diethoxy-phosphorylmethyl)-6,7,9,10,12,13,15,16-octahydro-5,8,11,14,17-pentaoxa-dibenzo[a,e $]$ cyclononadecene-3-ylmethyl]-phosphonic acid diethyl ester (8b). Preparation according to 8a. Yield: $3.15 \mathrm{~g}(52 \%)$ obtained from $5.00 \mathrm{~g}(8.99 \mathrm{mmol})$ of $\mathbf{7 b}$, colorless solid, mp $122{ }^{\circ} \mathrm{C} .{ }^{1} \mathrm{H}$ NMR $\left(\mathrm{CDCl}_{3}\right): \delta 1.22\left(\mathrm{t},{ }^{3} J 7.3 \mathrm{~Hz}, 12 \mathrm{H}, \mathrm{CH}_{3}\right), 3.10\left(\mathrm{~d},{ }^{2} J(\mathrm{P}, \mathrm{H})\right.$ $\left.21.5 \mathrm{~Hz}, 4 \mathrm{H}, \mathrm{CH}_{2} \mathrm{P}\right), 3.62-3.72\left(\mathrm{~m}, 8 \mathrm{H}, \mathrm{OCH}_{2}\right), 3.90-4.07\left(\mathrm{~m}, 12 \mathrm{H}, \mathrm{POCH}_{2}\right.$ and $\left.\mathrm{OCH}_{2}\right)$, 4.114.21 (m, 4H, $\left.\mathrm{OCH}_{2}\right), 6.78-6.88$ (m, 4H, H-2, H-4, H-16, H-20), 7.31 (d, 2H, ${ }^{3} J 7.3 \mathrm{~Hz}, 2 \mathrm{H}, \mathrm{H}-1$, $\mathrm{H}-21), 7.33$ (s, 2H, H-22, H-23). ${ }^{13} \mathrm{C}$ NMR $\left(\mathrm{CDCl}_{3}\right): \delta 16.3\left(\mathrm{CH}_{3}\right), 33.8\left(\mathrm{~d},{ }^{1} J(\mathrm{P}, \mathrm{C}) 137.3 \mathrm{~Hz}\right.$, $\left.\mathrm{CH}_{2} \mathrm{P}\right), 62.2\left(\mathrm{POCH}_{2}\right), 67.8,69.8,71.2,71.5\left(\mathrm{OCH}_{2}\right), 113.5,122.3,126.9,128.9$ (aromat. and olefin. CH), 126.3, 131.6, 156.4 (aromat. $\mathrm{C}_{\mathrm{q}}$ ). MS (FD), $\mathrm{m} / \mathrm{z}(\%) 670$ (100) [M] ${ }^{+}$. Anal. Calcd for: $\mathrm{C}_{32} \mathrm{H}_{48} \mathrm{O}_{11} \mathrm{P}_{2}$ (670.6): C, 57.31; H, 7.21. Found: C, 57.66; H, 7.06.

(E)-6,7,9,10-Tetrahydro-5,8,11-trioxa-dibenzo[a,e ]cyclo tridecene-3,13-dicarbaldehyde (9a). $\mathrm{Na}(450 \mathrm{mg}, 19.17 \mathrm{mmol})$ was dissolved in $50 \mathrm{~mL}$ of dry methanol before 2-nitropropane (1.80 $\mathrm{g}, 19.17 \mathrm{mmol})$ was slowly added. After a reaction time of $30 \mathrm{~min}$ dibromide $7 \mathbf{a}(3.00 \mathrm{~g}, 6.39$ mmol), suspended in $10 \mathrm{~mL}$ of dry methanol, was added in small portions. The mixture was stirred at room temperature in the dark for $48 \mathrm{~h}$. Then 1-2 mL of water was added in order to quench the process. The formed precipitate was filtered off and recrystallized from ethyl acetate. Yield: $1.17 \mathrm{~g}(54 \%)$. Yellowish powder, mp $165{ }^{\circ} \mathrm{C} .{ }^{1} \mathrm{H}$ NMR $\left(\mathrm{CDCl}_{3}\right): \delta$ 3.89-4.00 (m, 4H, $\mathrm{OCH}_{2}$ ), 4.20-4.29 (m, 4H, $\left.\mathrm{OCH}_{2}\right), 7.49$ (s, 2H, H-4, H-12), 7.53 (d, ${ }^{3} J$ 8.5 Hz, 2H, H-2, H-14), 7.65 (d, $\left.{ }^{3} \mathrm{~J} 8.5 \mathrm{~Hz}, 2 \mathrm{H}, \mathrm{H}-1, \mathrm{H}-6\right), 8.01$ (s, 2H, H-16, H-17), 9.93 (s, 2H, CHO). ${ }^{13} \mathrm{C}$ NMR $\left(\mathrm{CDCl}_{3}\right): \delta$ 69.8, $70.8\left(\mathrm{OCH}_{2}\right), 116.3,125.3,128.7,128.9$ (aromat. and olefin. $\left.\mathrm{CH}\right), 135.0$, 136.4, 157.8 (aromat. $\mathrm{C}_{\mathrm{q}}$ ), 191.3 (CHO). MS (FD), $\mathrm{m} / z(\%) 338$ (100) $[\mathrm{M}]^{+}$. Anal. Calcd for $\mathrm{C}_{20} \mathrm{H}_{18} \mathrm{O}_{5}$ (338.4): C, 71.00; H, 5.36. Found: C, 70.65; H, 5.60.

\section{(E)-6,7,9,10,12,13,15,16-Octahydro-5,8,11,14,17-pentaoxa-dibenzo[a,e]cyclononadecene-}

3,19-dicarbaldehyde (9b). Preparation according to 9a. Yield: $2.70 \mathrm{~g}$ (71\%) obtained from 5.00 g ( $8.99 \mathrm{mmol}) \mathbf{7 b}$. Yellowish crystals, mp $155{ }^{\circ} \mathrm{C} .{ }^{1} \mathrm{H}$ NMR $\left(\mathrm{CDCl}_{3}\right): \delta 3.69\left(\mathrm{~s}, 8 \mathrm{H}, \mathrm{OCH}_{2}\right)$, 400-4.09 (m, 4H, $\left.\mathrm{OCH}_{2}\right), 4.22-4.32\left(\mathrm{~m}, 4 \mathrm{H}, \mathrm{OCH}_{2}\right), 7.38$ (s, 2H, H-4, H-10), 7.44 (d, ${ }^{3} \mathrm{~J} 7.6 \mathrm{~Hz}$, 2H, H-2, H-20), 7.57 (s, 2H, H-22, H-23), 7.58 (d, $\left.{ }^{3} J 7.6 \mathrm{~Hz}, 2 \mathrm{H}, \mathrm{H}-1, \mathrm{H}-21\right), 9.93$ (s, 2H, $\mathrm{CHO}) .{ }^{13} \mathrm{C} \mathrm{NMR}\left(\mathrm{CDCl}_{3}\right): \delta$ 68.0, 69.5, 71.1, $71.5\left(\mathrm{OCH}_{2}\right), 110.4,124.2,129.5,129.5$ (aromat. and olefin. $\mathrm{CH}$ ), 133.3, 136.4, 157.1 (aromat. $\mathrm{C}_{\mathrm{q}}$ ), $191.5(\mathrm{CHO}) . \mathrm{MS}(\mathrm{FD}), m / z(\%)=426(100)$ $[\mathrm{M}]^{+}$. Anal. Calcd for $\mathrm{C}_{24} \mathrm{H}_{26} \mathrm{O}_{7}$ (426.4): C, 67.59; H, 6.15. Found: C, 67.47; H, 6.03.

General procedures for the preparation of the phenylenevinylene trimers with a stilbene crown (17a,b-23a,b). a) Diphosphonate 8a or $8 \mathbf{b}$ (2.0 mmol), aldehyde 10-16 (6.0 mmol), 550 mg Aliquat 336 and $1.0 \mathrm{~g}(15 \mathrm{mmol}) 85 \% \mathrm{KOH}$ powder $\left(15 \% \mathrm{H}_{2} \mathrm{O}\right)$ were heated in $100 \mathrm{~mL}$ of benzene $/ 5 \mathrm{~mL} \mathrm{H}_{2} \mathrm{O}$ to reflux for $24 \mathrm{~h}$. After cooling to ambient temperature, $30 \mathrm{~mL}$ of $\mathrm{H}_{2} \mathrm{O}$ were added. The organic phase was dried $\left(\mathrm{Na}_{2} \mathrm{SO}_{4}\right)$ and the volatile parts evaporated. The residue was dissolved in $\mathrm{CH}_{2} \mathrm{Cl}_{2}$ or $\mathrm{CHCl}_{3}$ and the solution dropped into $n$-hexane. Yellow crystals precipitated, which were analytically pure or could be recrystallized from diethylether. 
b) Dialdehyde 9a or 9b (1.0 mmol), phosphonate $24^{43,44}$ (1950 mg, $\left.2.5 \mathrm{mmol}\right), 500 \mathrm{mg}$ Aliquat 336 and $500 \mathrm{mg}(7.5 \mathrm{mmol}) 85 \% \mathrm{KOH}$ were heated in $200 \mathrm{~mL}$ of benzene/ $1 \mathrm{~mL}$ of $\mathrm{H}_{2} \mathrm{O}$ to reflux for $24 \mathrm{~h}$. After cooling to ambient temperature the reaction mixture was treated with $50 \mathrm{~mL}$ of $\mathrm{H}_{2} \mathrm{O}$. The organic phase was dried $\left(\mathrm{Na}_{2} \mathrm{SO}_{4}\right)$ and evaporated. Column chromatography $(40 \times 3 \mathrm{~cm}$ $\mathrm{SiO}_{2}$, diethylether/petroleum ether 1:2) yielded yellow crystals.

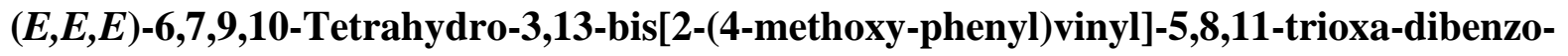
[a,e]cyclotridecene (17a). Method a), yield: $536 \mathrm{mg}(49 \%), \mathrm{mp} 254{ }^{\circ} \mathrm{C} .{ }^{1} \mathrm{H} \mathrm{NMR}\left(\mathrm{CDCl}_{3}\right): \delta$ $3.81\left(\mathrm{~s}, 6 \mathrm{H}, \mathrm{OCH}_{3}\right), 3.93-3.96\left(\mathrm{~m}, 4 \mathrm{H}, \mathrm{OCH}_{2}\right), 4.21-4.25\left(\mathrm{~m}, 4 \mathrm{H}, \mathrm{OCH}_{2}\right), 6.88 / 7.43\left(\mathrm{AA}^{\prime} \mathrm{BB}^{\prime}\right.$, $4 \mathrm{H}$, aromat. H), 6.91/7.04 (AB, ${ }^{3} J 16.4 \mathrm{~Hz}, 4 \mathrm{H}$, olefin. H), 7.11 (s, 2H, H-4, H-12), 7.16 (d, ${ }^{3} J$ $8.3 \mathrm{~Hz}, 2 \mathrm{H}, \mathrm{H}-2, \mathrm{H}-14), 7.46$ (d, $\left.{ }^{3} J 8.3 \mathrm{~Hz}, 2 \mathrm{H}, \mathrm{H}-1, \mathrm{H}-15\right), 7.82$ (s, 2H, H-16, H-17). ${ }^{13} \mathrm{C}$ NMR $\left(\mathrm{CDCl}_{3}\right): \delta 55.2\left(\mathrm{OCH}_{3}\right), 70.6,71.4\left(\mathrm{OCH}_{2}\right), 114.6,115.2,121.3,126.3,126.5,128.2,128.5$, 128.6 (aromat. and olefin. $\mathrm{CH}), 128.9,130.4,138.7,158.1,159.8$ (aromat. $\mathrm{C}_{\mathrm{q}}$ ).MS (FD), $\mathrm{m} / z(\%)$ $=546(100)[\mathrm{M}]^{+} \cdot \mathrm{UV} / \mathrm{Vis}\left(\mathrm{CH}_{2} \mathrm{Cl}_{2}\right): \lambda_{\max }(\varepsilon)=401 \mathrm{~nm}\left(56700 \mathrm{M}^{-1} \mathrm{~cm}^{-1}\right)$. Anal. Calcd for $\mathrm{C}_{36} \mathrm{H}_{34} \mathrm{O}_{5}$ (546.7): C, 79.10; H, 6.27. Found: C, 79.20; H, 6.28.

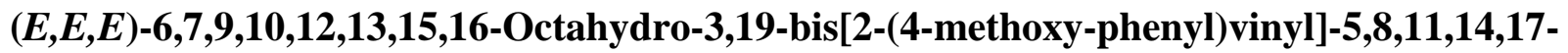
pentaoxa-dibenzo[a,e]cyclononadecene (17b). Method a), yield: $837 \mathrm{mg}(66 \%), \mathrm{mp} 226-233$ ${ }^{\circ} \mathrm{C} .{ }^{1} \mathrm{H}$ NMR $\left(\mathrm{CDCl}_{3}\right): \delta$ 3.68-3.72 $\left(\mathrm{m}, 8 \mathrm{H}, \mathrm{OCH}_{2}\right), 3.82\left(\mathrm{~s}, 6 \mathrm{H}, \mathrm{OCH}_{3}\right), 4.02-4.09(\mathrm{~m}, 4 \mathrm{H}$, $\left.\mathrm{OCH}_{2}\right), 4.22-4.30\left(\mathrm{~m}, 4 \mathrm{H}, \mathrm{OCH}_{2}\right), 6.88 / 7.44\left(\mathrm{AA}^{\prime} \mathrm{BB}^{\prime}, 4 \mathrm{H}\right.$, aromat. H), 6.91/7.05 (AB, ${ }^{3} J 16.4$ Hz, 4H, olefin. H), 7.01 (s, 2H, H-4, H-18), 7.08 (d, ${ }^{3} J$ 7.3 Hz, 2H, H-2, H-20), 7.38-7.43 (m, $2 \mathrm{H}, \mathrm{H}-1, \mathrm{H}-21), 7.42$ (s, 2H, H-22, H-23). ${ }^{13} \mathrm{C} \mathrm{NMR}\left(\mathrm{CDCl}_{3}\right): \delta 55.3\left(\mathrm{OCH}_{3}\right), 68.0,70.0,71.2$, 71.7, $\left(\mathrm{OCH}_{2}\right), 109.9,114.2,119.3,126.5,126.7,127.7,128.1,128.8$ (aromat. and olefin. $\mathrm{CH}$ ), $127.1,130.2,137.8,156.9,159.4$ (aromat. $\mathrm{C}_{\mathrm{q}}$ ). MS (FD), $m / z(\%)=634(100)[\mathrm{M}]^{+}$. UV/Vis $\left(\mathrm{CH}_{2} \mathrm{Cl}_{2}\right): \lambda_{\max }(\varepsilon)=401 \mathrm{~nm}\left(54800 \mathrm{M}^{-1} \mathrm{~cm}^{-1}\right)$. Anal. Calcd for $\mathrm{C}_{40} \mathrm{H}_{42} \mathrm{O}_{7}$ (634.8): C, 75.69; $\mathrm{H}$, 6.67. Found: C, 74.91; H, 6.22 (incomplete combustion).

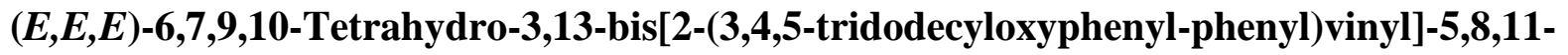
trioxa-dibenzo[a,e]cyclotridecene (18a). Method a), yield: $1620 \mathrm{mg}(51 \%)$; method b), yield: $1003 \mathrm{mg}(63 \%)$; mp $91{ }^{\circ} \mathrm{C}$. ${ }^{1} \mathrm{H} \mathrm{NMR}\left(\mathrm{CDCl}_{3}\right): \delta \quad 0.84-0.90\left(\mathrm{~m}, 18 \mathrm{H}, \mathrm{CH}_{3}\right), 1.20-1.38(\mathrm{~m}, 96 \mathrm{H}$, $\left.\mathrm{CH}_{2}\right), 1.40-1.50\left(\mathrm{~m}, 12 \mathrm{H}, \mathrm{CH}_{2}\right), 1.68-1.75\left(\mathrm{~m}, 4 \mathrm{H}, \mathrm{CH}_{2}\right), 1.75-1.84\left(\mathrm{~m}, 8 \mathrm{H}, \mathrm{CH}_{2}\right), 3.93-3.98(\mathrm{~m}$, $\left.8 \mathrm{H}, \mathrm{OCH}_{2}\right), 3.98-4.03\left(\mathrm{~m}, 8 \mathrm{H}, \mathrm{OCH}_{2}\right), 4.20-4.24\left(\mathrm{~m}, 4 \mathrm{H}, \mathrm{OCH}_{2}\right), 6.69(\mathrm{~s}, 4 \mathrm{H}$, aromat. H), 6.91/6.98 (AB, ${ }^{3} J 16.2 \mathrm{~Hz}, 4 \mathrm{H}$, olefin. H), 7.11 (s, 2H, H-4, H-12), 7.16 (d, ${ }^{3} J 8.1 \mathrm{~Hz}, 2 \mathrm{H}, \mathrm{H}-2$, $\mathrm{H}-14), 7.46$ (d, $\left.{ }^{3} J \quad 8.1 \mathrm{~Hz}, 2 \mathrm{H}, \mathrm{H}-1, \mathrm{H}-15\right), 7.82$ (s, 2H, H-16, H-17). ${ }^{13} \mathrm{C} \mathrm{NMR}\left(\mathrm{CDCl}_{3}\right): \delta 14.0$ $\left(\mathrm{CH}_{3}\right), 22.6,26.1,29.3,29.4,29.5,29.6,29.7,30.3,31.8\left(\mathrm{CH}_{2}\right.$, partly superimposed), 69.3, 70.3, 70.9, $73.5\left(\mathrm{OCH}_{2}\right), 105.6,115.0,121.1,126.2,127.3,128.8,128.9$ (aromat. and olefin. $\mathrm{CH}$ ), 128.1, 132.5, 137.5, 138.7, 153.4, 157.6 (aromat. $\mathrm{C}_{\mathrm{q}}$ ). MS (FD), $m / z(\%)=1592(100)[\mathrm{M}]^{+}$. $\mathrm{UV} / \mathrm{Vis}\left(\mathrm{CH}_{2} \mathrm{Cl}_{2}\right): \lambda_{\max }(\varepsilon)=406 \mathrm{~nm}\left(55400 \mathrm{M}^{-1} \mathrm{~cm}^{-1}\right)$. Anal. Calcd for $\mathrm{C}_{106} \mathrm{H}_{174} \mathrm{O}_{9}$ (1592.5): $\mathrm{C}$, 79.95; H, 11.01. Found: C, 79.77; H, 10.88. 


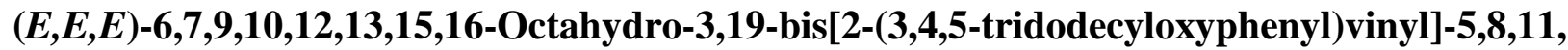
14,17-pentaoxa-dibenzo[a,e]cyclononadecene (18b). Method a), yield: $1850 \mathrm{mg}$ (55\%); method b), yield: $825 \mathrm{mg}(49 \%)$; mp $87{ }^{\circ} \mathrm{C} .{ }^{1} \mathrm{H}$ NMR $\left(\mathrm{CDCl}_{3}\right): \delta \quad 0.84-0.91\left(\mathrm{~m}, 18 \mathrm{H}, \mathrm{CH}_{3}\right)$, 1.20-1.39 (m, 96H, $\left.\mathrm{CH}_{2}\right), 1.42-1.51\left(\mathrm{~m}, 12 \mathrm{H}, \mathrm{CH}_{2}\right), 1.69-1.76\left(\mathrm{~m}, 4 \mathrm{H}, \mathrm{CH}_{2}\right), 1.76-1.85(\mathrm{~m}, 8 \mathrm{H}$, $\left.\mathrm{CH}_{2}\right), 3.69-3.73\left(\mathrm{~m}, 4 \mathrm{H}, \mathrm{OCH}_{2}\right), 3.74-3.78\left(\mathrm{~m}, 4 \mathrm{H}, \mathrm{OCH}_{2}\right), 3.93-3.98\left(\mathrm{~m}, 4 \mathrm{H}, \mathrm{OCH}_{2}\right), 3.98-4.04$ $\left(\mathrm{m}, 8 \mathrm{H}, \mathrm{OCH}_{2}\right), 4.04-4.08\left(\mathrm{~m}, 4 \mathrm{H}, \mathrm{OCH}_{2}\right), 4.23-4.28\left(\mathrm{~m}, 4 \mathrm{H}, \mathrm{OCH}_{2}\right), 6.70(\mathrm{~s}, 4 \mathrm{H}$, aromat. $\mathrm{H})$, 6.92/6.98 (AB, ${ }^{3} J$ 16.3 Hz, 4H, olefin. H), 7.01 (s, 2H, H-4, H-18), 7.08 (d, ${ }^{3} J$ 7.8 Hz, 2H, H-2, $\mathrm{H}-20), 7.40$ (d, $\left.{ }^{3} J 7.8 \mathrm{~Hz}, 2 \mathrm{H}, \mathrm{H}-1, \mathrm{H}-21\right), 7.44$ (s, 2H, H-22, H-23). ${ }^{13} \mathrm{C} \mathrm{NMR}\left(\mathrm{CDCl}_{3}\right): \delta 14.0$ $\left(\mathrm{CH}_{3}\right)$, 22.6, 26.1, 29.3, 29.4, 29.5, 29.6, 29.7, 29.8, 30.3, $31.9\left(\mathrm{CH}_{2}\right.$, partly superimposed), 67.9, 69.3, 70.0, 71.2, 71.7, $73.5\left(\mathrm{OCH}_{2}\right), 105.5,109.9,119.5,126.8,127.5,128.8,129.0$ (aromat. and olefin. CH), 127.2, 132.5, 137.5, 138.7, 153.4, 156.8 (aromat. $\mathrm{C}_{\mathrm{q}}$ ). MS (FD), $\mathrm{m} / z(\%)=1680$ (100) $[\mathrm{M}]^{+}$. UV/Vis $\left(\mathrm{CH}_{2} \mathrm{Cl}_{2}\right): \lambda_{\max }(\varepsilon)=406 \mathrm{~nm}\left(59500 \mathrm{M}^{-1} \mathrm{~cm}^{-1}\right)$. Anal. Calcd for $\mathrm{C}_{110} \mathrm{H}_{182} \mathrm{O}_{11}$ (1680.7): C, 78.61; H, 10.92. Found: C, 78.70; H, 10.53.

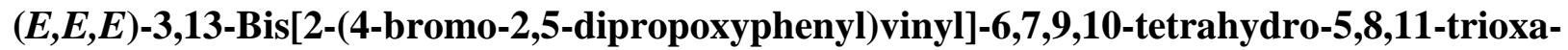
dibenzo[a,e $]$ cyclotridecene (19a). Method a), yield: $1120 \mathrm{mg}(64 \%), \mathrm{mp} 188{ }^{\circ} \mathrm{C} .{ }^{1} \mathrm{H}$ NMR $\left(\mathrm{CDCl}_{3}\right): \delta$ 1.04-1.12 (m, 12H, $\left.\mathrm{CH}_{3}\right), 1.80-1.90\left(\mathrm{~m}, 8 \mathrm{H}, \mathrm{CH}_{2}\right), 3.90-3.94\left(\mathrm{~m}, 4 \mathrm{H}, \mathrm{OCH}_{2}\right)$, 3.94$3.96\left(\mathrm{~m}, 4 \mathrm{H}, \mathrm{OCH}_{2}\right), 3.97-4.01\left(\mathrm{~m}, 4 \mathrm{H}, \mathrm{OCH}_{2}\right), 4.20-4.25\left(\mathrm{~m}, 4 \mathrm{H}, \mathrm{OCH}_{2}\right), 7.05 / 7.36\left(\mathrm{AB},{ }^{3} J\right.$ $16.4 \mathrm{~Hz}, 4 \mathrm{H}$, olefin. H), 7.07 (s, 2H, aromat. H), 7.10 (s, 2H, aromat. H), 7.12 (s, 2H, H-4, H-12), 7.19 (d, $\left.{ }^{3} \mathrm{~J} 8.2 \mathrm{~Hz}, 2 \mathrm{H}, \mathrm{H}-2, \mathrm{H}-14\right), 7.47$ (d, $\left.{ }^{3} \mathrm{~J} 8.2 \mathrm{~Hz}, 2 \mathrm{H}, \mathrm{H}-1, \mathrm{H}-15\right), 7.84$ (s, 2H, H-16, H17). ${ }^{13} \mathrm{C} \mathrm{NMR}\left(\mathrm{CDCl}_{3}\right): \delta 10.5,10.5\left(\mathrm{CH}_{3}\right), 22.6,22.6\left(\mathrm{CH}_{2}\right), 70.2,70.9,71.2,71.8\left(\mathrm{OCH}_{2}\right)$, 111.9, 115.1, 118.1, 121.1, 123.0, 126.2, 128.1, 128.9 (aromat. and olefin. CH), 112.0, 126.6, 137.7, 149.9, 151.1, 156.8 (aromat. $\mathrm{C}_{\mathrm{q}}$, partly superimposed). $\mathrm{MS}(\mathrm{FD}), \mathrm{m} / \mathrm{z}(\%)=878,876,874$ (100) $[\mathrm{M}]^{+}, \mathrm{Br}_{2}$ isotope pattern. UV/Vis $\left(\mathrm{CH}_{2} \mathrm{Cl}_{2}\right): \lambda_{\max }(\varepsilon)=412 \mathrm{~nm}\left(58300 \mathrm{M}^{-1} \mathrm{~cm}^{-1}\right)$. Anal. Calcd for $\mathrm{C}_{46} \mathrm{H}_{52} \mathrm{O}_{7} \mathrm{Br}_{2}$ (876.7): C, 63.02; H, 5.98; Br, 18.23. Found: C, 62.84; H, 5.90; Br, 18.05 .

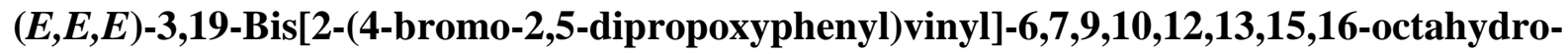
$\mathbf{5 , 8 , 1 1 , 1 4 , 1 7 - p e n t a o x a - d i b e n z o [ a , e ] c y c l o n o n a d e c e n e ~ ( 1 9 b ) . ~ M e t h o d ~ a ) , ~ y i e l d : ~} 1100 \mathrm{mg}(57 \%)$. ${ }^{1} \mathrm{H}$ NMR $\left(\mathrm{CDCl}_{3}\right): \delta$ 1.05-1.11 $\left(\mathrm{m}, 12 \mathrm{H}, \mathrm{CH}_{3}\right), 1.80-1.90\left(\mathrm{~m}, 8 \mathrm{H}, \mathrm{CH}_{2}\right), 3.69-3.73(\mathrm{~m}, 4 \mathrm{H}$, $\left.\mathrm{OCH}_{2}\right), 3.73-3.77\left(\mathrm{~m}, 4 \mathrm{H}, \mathrm{OCH}_{2}\right), 3.90-3.94\left(\mathrm{~m}, 4 \mathrm{H}, \mathrm{OCH}_{2}\right), 3.98-4.02\left(\mathrm{~m}, 4 \mathrm{H}, \mathrm{OCH}_{2}\right), 4.02-$ $4.07\left(\mathrm{~m}, 4 \mathrm{H}, \mathrm{OCH}_{2}\right), 4.23-4.27\left(\mathrm{~m}, 4 \mathrm{H}, \mathrm{OCH}_{2}\right), 7.01$ (s, 2H, aromat. H), 7.07/7.36 (AB, ${ }^{3} J 16.4$ $\mathrm{Hz}, 4 \mathrm{H}$, olefin. H), 7.07 (s, 2H, aromat. H), 7.11 (s, 2H, H-4, H-18), 7.12 (d, ${ }^{3} J 7.9 \mathrm{~Hz}, \mathrm{H}-2, \mathrm{H}-$ 20), 7.42 (d, $\left.{ }^{3} J 7.9 \mathrm{~Hz}, 2 \mathrm{H}, \mathrm{H}-1, \mathrm{H}-21\right), 7.45$ (s, 2H, H-22, H-23). ${ }^{13} \mathrm{C} \mathrm{NMR}\left(\mathrm{CDCl}_{3}\right): \delta 10.6$, $10.6\left(\mathrm{CH}_{3}\right), 22.7,22.7\left(\mathrm{CH}_{2}\right), 68.0,70.0,71.2,71.3,71.6,71.9\left(\mathrm{OCH}_{2}\right), 110.2,111.9,118.2$, 119.5, 123.0, 126.9, 129.0, 129.3 (aromat. and olefin. $\mathrm{CH}$ ), 112.0, 126.8, 127.4, 137.8, 150.0, 151.2, 156.9 (aromat. $\mathrm{C}_{\mathrm{q}}$ ). MS (FD), $m / z(\%)=966,964,962(100)[\mathrm{M}]^{+}, \mathrm{Br}_{2}$ isotope pattern. UV/Vis $\left(\mathrm{CH}_{2} \mathrm{Cl}_{2}\right): \lambda_{\max }(\varepsilon)=412 \mathrm{~nm}\left(69900 \mathrm{M}^{-1} \mathrm{~cm}^{-1}\right)$. Anal. Calcd for $\mathrm{C}_{50} \mathrm{H}_{60} \mathrm{O}_{9} \mathrm{Br}_{2}$ (964.8): $\mathrm{C}$, 62.24; H, 6.27; Br, 16.56. Found: C, 61.96; H, 5.89; Br, 16.42. 


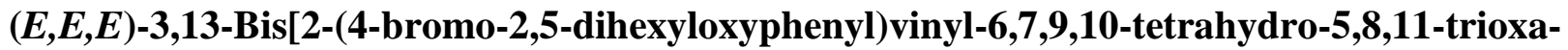
dibenzo[a,e]cyclotridecene (20a). Method a), yield: $1023 \mathrm{mg}(49 \%) .{ }^{1} \mathrm{H} \mathrm{NMR}\left(\mathrm{CDCl}_{3}\right): \delta$ 0.87-0.93 (m, 12H, $\left.\mathrm{CH}_{3}\right), 1.25-1.40\left(\mathrm{~m}, 16 \mathrm{H}, \mathrm{CH}_{2}\right), 1.46-1.55\left(\mathrm{~m}, 8 \mathrm{H}, \mathrm{CH}_{2}\right), 1.78-1.86(\mathrm{~m}, 8 \mathrm{H}$, $\left.\mathrm{CH}_{2}\right), 3.92-3.97\left(\mathrm{~m}, 8 \mathrm{H}, \mathrm{OCH}_{2}\right), 3.99-4.03\left(\mathrm{~m}, 4 \mathrm{H}, \mathrm{OCH}_{2}\right), 4.20-4.23\left(\mathrm{~m}, 4 \mathrm{H}, \mathrm{OCH}_{2}\right), 7.05 / 7.35$ $\left(\mathrm{AB},{ }^{3} \mathrm{~J} 16.4 \mathrm{~Hz}, 4 \mathrm{H}\right.$, olefin. H), 7.06 (s, 2H, aromat. H), 7.09 (s, 2H, aromat. H), 7.12 (s, 2H, H4, H-12), 7.19 (d, ${ }^{3} J$ 8.0 Hz, 2H, H-2, H-14), 7.47 (d, ${ }^{3} J$ 8.0 Hz, 2H, H-1, H-15), 7.84 (s, 2H, H16, H-17). ${ }^{13} \mathrm{C}$ NMR $\left(\mathrm{CDCl}_{3}\right): \delta 13.9,13.9\left(\mathrm{CH}_{3}\right), 22.5,22.6,25.6,25.8,29.5,29.5,31.5,31.5$ $\left(\mathrm{CH}_{2}\right), 69.6,70.2,70.3,70.8\left(\mathrm{OCH}_{2}\right), 111.8,115.1,117.9,121.2,123.0,126.2,128.1,128.4$ (aromat. and olefin. CH), 112.0, 126.6, 137.7, 149.9, 151.1, 157.1 (aromat. $\mathrm{C}_{\mathrm{q}}$, partly superimposed). MS (FD), $m / z(\%)=1042,1044,1046(100)[\mathrm{M}]^{+}, \mathrm{Br}_{2}$ isotope pattern. UV/Vis $\left(\mathrm{CH}_{2} \mathrm{Cl}_{2}\right): \lambda_{\max }(\varepsilon)=412 \mathrm{~nm}\left(67500 \mathrm{M}^{-1} \mathrm{~cm}^{-1}\right)$. Anal. Calcd for $\mathrm{C}_{58} \mathrm{H}_{76} \mathrm{O}_{7} \mathrm{Br}_{2}$ (1045.1): C, 66.66; H, 7.33; Br, 15.29. Found: C, 66.27; H, 7.08; Br, 15.1.

(E,E,E)-3,19-Bis[2-(4-bromo-2,5-dihexyloxyphenyl)vinyl]-6,7,9,10,12,13,15,16-octahydro5,8,11,14,17-pentaoxa-dibenzo[a,e]cyclononadecene (20b). Method a), yield: $975 \mathrm{mg}$ (43\%), mp $120{ }^{\circ} \mathrm{C} .{ }^{1} \mathrm{H}$ NMR $\left(\mathrm{CDCl}_{3}\right): \delta$ 0.84-0.96 (m, $\left.12 \mathrm{H}, \mathrm{CH}_{3}\right), 1.25-1.40\left(\mathrm{~m}, 16 \mathrm{H}, \mathrm{CH}_{2}\right), 1.45-1.58$ $\left(\mathrm{m}, 8 \mathrm{H}, \mathrm{CH}_{2}\right), 1.75-1.88\left(\mathrm{~m}, 8 \mathrm{H}, \mathrm{CH}_{2}\right), 3.67-3.78\left(\mathrm{~m}, 8 \mathrm{H}, \mathrm{OCH}_{2}\right), 3.90-4.00\left(\mathrm{~m}, 4 \mathrm{H}, \mathrm{OCH}_{2}\right)$, 4.00-4.09 (m, 8H, $\left.\mathrm{OCH}_{2}\right), 4.20-4.29\left(\mathrm{~m}, 4 \mathrm{H}, \mathrm{OCH}_{2}\right), 7.01(\mathrm{~s}, 2 \mathrm{H}$, aromat. $\mathrm{H}), 7.06$ (s, 2H, aromat. H), 7.07/7.35 (AB, ${ }^{3} J 16.4 \mathrm{~Hz}, 4 \mathrm{H}$, olefin. H), 7.10 (s, 2H, H-4, H-18), 7.13 (d, ${ }^{3} J 7.9$ $\mathrm{Hz}, 2 \mathrm{H}, \mathrm{H}-2, \mathrm{H}-20), 7.42$ (d, $\left.{ }^{3} \mathrm{~J} 7.9 \mathrm{~Hz}, 2 \mathrm{H}, \mathrm{H}-1, \mathrm{H}-21\right), 7.45$ (s, 2H, H-22, H-23). ${ }^{13} \mathrm{C}$ NMR $\left(\mathrm{CDCl}_{3}\right): \delta 14.0\left(\mathrm{CH}_{3}\right), 22.6,25.7,25.8,29.3,31.5\left(\mathrm{CH}_{2}\right.$, partly superimposed $), 68.0,69.7,70.0$, $70.4,71.2,71.7\left(\mathrm{OCH}_{2}\right), 110.2,111.9,118.0,119.5,123.1,126.9,129.0,129.3$ (aromat. and olefin. $\mathrm{CH}), 112.0,126.7,127.4,137.8,150.0,151.2,156.9$ (aromat. $\left.\mathrm{C}_{\mathrm{q}}\right) . \mathrm{MS}(\mathrm{FD}), \mathrm{m} / z(\%)=$ 1130, 1132, $1134(100)[\mathrm{M}]^{+}, \mathrm{Br}_{2}$ isotope pattern. UV/Vis $\left(\mathrm{CH}_{2} \mathrm{Cl}_{2}\right): \lambda_{\max }(\varepsilon)=412 \mathrm{~nm}(59100$ $\mathrm{M}^{-1} \mathrm{~cm}^{-1}$ ). Anal. Calcd for $\mathrm{C}_{62} \mathrm{H}_{84} \mathrm{O}_{9} \mathrm{Br}_{2}$ (1133.2): C, 65.72; H, 7.47; Br, 14.10. Found: C, 65.83; $\mathrm{H}, 7.13 ; \mathrm{Br}, 13.90$.

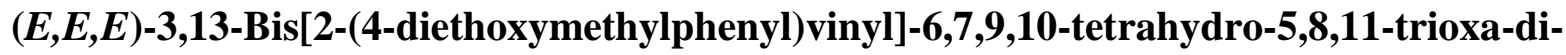
benzo[a,e]cyclotridecene (21a). Method a), yield: $770 \mathrm{mg}(56 \%)$, mp $204{ }^{\circ} \mathrm{C} .{ }^{1} \mathrm{H}$ NMR $\left(\mathrm{CDCl}_{3}\right): \delta 1.23\left(\mathrm{t},{ }^{3} \mathrm{~J} 7.4 \mathrm{~Hz}, 12 \mathrm{H}, \mathrm{CH}_{3}\right), 3.49-3.66\left(\mathrm{~m}, 8 \mathrm{H}, \mathrm{OCH}_{2} \mathrm{CH}_{3}\right), 3.91-3.98(\mathrm{~m}, 4 \mathrm{H}$, $\left.\mathrm{OCH}_{2}\right), 4.20-4.25\left(\mathrm{~m}, 4 \mathrm{H}, \mathrm{OCH}_{2}\right), 5.50\left(\mathrm{~s}, 2 \mathrm{H}, \mathrm{CH}\left(\mathrm{OC}_{2} \mathrm{H}_{5}\right)_{2}\right), 7.05 / 7.09\left(\mathrm{AB},{ }^{3} \mathrm{~J} 16.4 \mathrm{~Hz}, 4 \mathrm{H}\right.$, olefin. H), 7.13 (s, 2H, H-4, H-12), 7.18 (d, ${ }^{3} J$ 8.2 Hz, 2H, H-2, H-14), 7.44/7.48 (AA'BB', 8H, aromat. H), 7.48 (d, $\left.{ }^{3} J 8.2 \mathrm{~Hz}, 2 \mathrm{H}, \mathrm{H}-1, \mathrm{H}-15\right), 7.84$ (s, 2H, H-16, H-17). ${ }^{13} \mathrm{C}$ NMR $\left(\mathrm{CDCl}_{3}\right): \delta$ $15.2\left(\mathrm{CH}_{3}\right), 61.1\left(\mathrm{OCH}_{2} \mathrm{CH}_{3}\right), 70.3,71.0\left(\mathrm{OCH}_{2}\right), 101.4\left(\mathrm{CH}\left(\mathrm{OC}_{2} \mathrm{H}_{5}\right)_{2}\right), 115.2,121.3,126.3$, 126.4, 127.3, 128.2, 128.4, 128.6 (aromat. and olefin. $\mathrm{CH}), 129.1,137.4,137.4,138.7,157.7$ (aromat. $\mathrm{C}_{\mathrm{q}}$ ). MS (FD), $\mathrm{m} / z(\%)=690(100)[\mathrm{M}]^{+}$. UV/Vis $\left(\mathrm{CH}_{2} \mathrm{Cl}_{2}\right): \lambda_{\max }(\varepsilon)=399 \mathrm{~nm}(67300$ $\mathrm{M}^{-1} \mathrm{~cm}^{-1}$ ). Anal. Calcd for $\mathrm{C}_{44} \mathrm{H}_{50} \mathrm{O}_{7}$ (690.9): C, 76.49; H, 7.29. Found: C, 76.19; H, 7.01.

$(E, E, E)-3,19-B i s[2-(4-d i e t h o x y m e t h y l p h e n y l) v i n y l]-6,7,9,10,12,13,15,16-o c t a h y d r o-5,8,11$, 14,17-pentaoxa-dibenzo[a,e]cyclononadecene (21b). Method a), yield: $970 \mathrm{mg}$ (62\%), mp 209 
${ }^{\circ} \mathrm{C} .{ }^{1} \mathrm{H}$ NMR $\left(\mathrm{CDCl}_{3}\right): \delta \quad 1.24\left(\mathrm{t},{ }^{3} \mathrm{~J} \quad 7.4 \mathrm{~Hz}, 12 \mathrm{H}, \mathrm{CH}_{3}\right), 3.46-3.67\left(\mathrm{~m}, 8 \mathrm{H}, \mathrm{OCH}_{2} \mathrm{CH}_{3}\right), 3.67-$ $3.79\left(\mathrm{~m}, 8 \mathrm{H}, \mathrm{OCH}_{2}\right), 4.01-4.10\left(\mathrm{~m}, 4 \mathrm{H}, \mathrm{OCH}_{2}\right), 4.22-4.30\left(\mathrm{~m}, 4 \mathrm{H}, \mathrm{OCH}_{2}\right), 5.50(\mathrm{~s}, 2 \mathrm{H}$, $\left.\mathrm{CH}\left(\mathrm{OC}_{2} \mathrm{H}_{5}\right)_{2}\right), 7.03$ (s, 2H, H-4, H-18), 7.08 (s, 4H, olefin. H), 7.11 (d, ${ }^{3} J 8.2 \mathrm{~Hz}, 2 \mathrm{H}, \mathrm{H}-2, \mathrm{H}-$ 20), 7.42 (d, ${ }^{3} J$ $\left.8.2 \mathrm{~Hz}, 2 \mathrm{H}, \mathrm{H}-1, \mathrm{H}-21\right), 7.44$ (s, 2H, H-22, H-23), 7.45/7.50 (AA'BB', 8H, aromat. H). ${ }^{13} \mathrm{C} \mathrm{NMR}\left(\mathrm{CDCl}_{3}\right): \delta 15.2\left(\mathrm{CH}_{3}\right), 61.0\left(\mathrm{OCH}_{2} \mathrm{CH}_{3}\right), 68.1,70.0,71.3,71.7\left(\mathrm{OCH}_{2}\right)$, $101.4\left(\mathrm{CH}\left(\mathrm{OC}_{2} \mathrm{H}_{5}\right)_{2}\right), 110.2,119.7,126.3,127.0,127.1,128.3,128.8,129.0$ (aromat. and olefin. $\mathrm{CH}), 127.5,137.4,137.5,138.7,156.9$ (aromat. $\mathrm{C}_{\mathrm{q}}$ ). MS (FD), $\mathrm{m} / z(\%)=778(100)[\mathrm{M}]^{+}$. UV $\left(\mathrm{CH}_{2} \mathrm{Cl}_{2}\right): \lambda_{\max }(\varepsilon)=399 \mathrm{~nm}\left(71400 \mathrm{M}^{-1} \mathrm{~cm}^{-1}\right)$. Anal. Calcd for $\mathrm{C}_{48} \mathrm{H}_{58} \mathrm{O}_{9}$ (779.0): C, 74.01; $\mathrm{H}$, 7.50. Found: C, 74.22; H, 7.39.

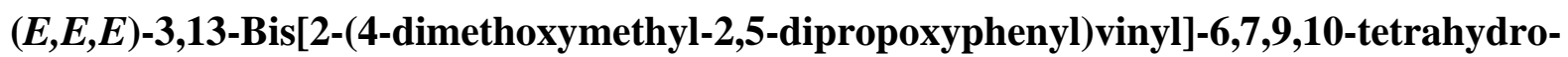
5,8,11-trioxa-dibenzo[a,e $]$ cyclotridecene (22a). Method a), yield: $830 \mathrm{mg}$ (48\%) yellow crystals, mp $112{ }^{\circ} \mathrm{C} .{ }^{1} \mathrm{H}$ NMR $\left(\mathrm{CDCl}_{3}\right): \delta$ 1.04-1.07 (m, 12H, $\left.\mathrm{CH}_{3}\right), 1.80-1.85\left(\mathrm{~m}, 8 \mathrm{H}, \mathrm{CH}_{2}\right)$, $3.39\left(\mathrm{~s}, 12 \mathrm{H}, \mathrm{OCH}_{3}\right), 3.95-3.99\left(\mathrm{~m}, 12 \mathrm{H}, \mathrm{OCH}_{2}\right), 4.20-4.22\left(\mathrm{~m}, 4-\mathrm{H}, \mathrm{OCH}_{2}\right), 5.62(\mathrm{~s}, 2 \mathrm{H}$, $\left.\mathrm{CH}\left(\mathrm{OCH}_{3}\right)_{2}\right), 7.06 / 7.44(\mathrm{AB}, 16.3 \mathrm{~Hz}, 4 \mathrm{H}$, olefin. $\mathrm{H}), 7.07$ (s, 4H, aromat. H), 7.13 (s, 2H, H-4, H-12), 7.20 (d, ${ }^{3}$ J $\left.8.1 \mathrm{~Hz}, 2 \mathrm{H}, \mathrm{H}-2, \mathrm{H}-14\right), 7.47$ (d, $\left.{ }^{3} \mathrm{~J} 8.0 \mathrm{~Hz}, 2 \mathrm{H}, \mathrm{H}-1, \mathrm{H}-15\right), 7.83$ (s, 2H, H16, H-17). ${ }^{13} \mathrm{C} \mathrm{NMR}\left(\mathrm{CDCl}_{3}\right): \delta 10.7\left(\mathrm{CH}_{3}\right), 22.8\left(\mathrm{CH}_{2}\right), 54.1\left(\mathrm{OCH}_{3}\right), 70.3,70.9,70.9,71.1$ $\left(\mathrm{OCH}_{2}\right), 99.6\left(\mathrm{CH}\left(\mathrm{OCH}_{3}\right)_{2}\right), 110.4,112.1,115.2,121.3,123.7,126.3,128.1,128.8$ (aromat. and olefin. $\mathrm{CH}), 127.3,127.5,128.9,138.0,150.9,150.9,157.6$ (aromat. $\mathrm{C}_{\mathrm{q}}$ ). $\mathrm{MS}(\mathrm{FD}), \mathrm{m} / \mathrm{z}(\%)=$ $866(100)[\mathrm{M}]^{+}$. UV/Vis $\left(\mathrm{CH}_{2} \mathrm{Cl}_{2}\right): \lambda_{\max }(\varepsilon)=411 \mathrm{~nm}\left(67000 \mathrm{M}^{-1} \mathrm{~cm}^{-1}\right)$. Anal. Calcd for $\mathrm{C}_{52} \mathrm{H}_{66} \mathrm{O}_{11}$ (867.1): C, 72.03; H, 7.67. Found: C, 71.63; H, 7.33.

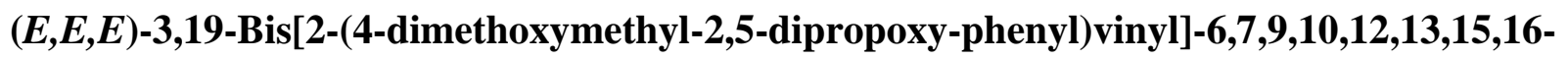
octahydro-5,8,11, 14,17-pentaoxa-dibenzo[a,e]cyclononadecene (22b). Method a), yield: 920 $\mathrm{mg}(48 \%)$ yellow crystals, mp $251-265{ }^{\circ} \mathrm{C} .{ }^{1} \mathrm{H} \mathrm{NMR}\left(\mathrm{CDCl}_{3}\right): \delta 1.04-1.07\left(\mathrm{~m}, 12 \mathrm{H}, \mathrm{CH}_{3}\right), 1.82-$ $1.87\left(\mathrm{~m}, 8 \mathrm{H}, \mathrm{CH}_{2}\right), 3.39\left(\mathrm{~s}, 12 \mathrm{H}, \mathrm{OCH}_{3}\right), 3.70-3.77\left(\mathrm{~m}, 8 \mathrm{H}, \mathrm{OCH}_{2}\right), 3.95-4.00\left(\mathrm{~m}, 8 \mathrm{H}, \mathrm{OCH}_{2}\right)$, 4.02-4.07 (m, 4H, $\left.\mathrm{OCH}_{2}\right), 4.23-4.27\left(\mathrm{~m}, 4 \mathrm{H}, \mathrm{OCH}_{2}\right), 5.62\left(\mathrm{~s}, 2 \mathrm{H}, \mathrm{CH}\left(\mathrm{OCH}_{3}\right)_{2}\right), 7.03(\mathrm{~s}, 2 \mathrm{H}, \mathrm{H}-4$, H-18), 7.07/7.43 (AB, ${ }^{3} J 16.4 \mathrm{~Hz}, 4 \mathrm{H}$, olefin. H), 7.08, 7.08 (2 s, 4H, aromat. H), 7.12 (d, ${ }^{3} J 8.1$ $\mathrm{Hz}, 2 \mathrm{H}, \mathrm{H}-2, \mathrm{H}-20), 7.41$ (d, $\left.{ }^{3} \mathrm{~J} 8.1 \mathrm{~Hz}, 2 \mathrm{H}, \mathrm{H}-1, \mathrm{H}-21\right), 7.44$ (s, 2H, H-22, H-23). ${ }^{13} \mathrm{C}$ NMR $\left(\mathrm{CDCl}_{3}\right): \delta 10.7\left(\mathrm{CH}_{3}\right), 22.9\left(\mathrm{CH}_{2}\right), 54.2\left(\mathrm{OCH}_{3}\right), 68.0,70.0,71.0,71.2,71.3,71.7\left(\mathrm{OCH}_{2}\right), 99.7$ $\left(\mathrm{CH}\left(\mathrm{OCH}_{3}\right)_{2}\right), 110.3,110.5,112.2,119.6,123.7,126.9,129.0,129.1$ (aromat. and olefin. $\left.\mathrm{CH}\right)$, $127.2,127.3,127.5,138.1,150.9,151.0,156.9$ (aromat. $\mathrm{C}_{\mathrm{q}}$ ). MS (FD), $m / z(\%)=955(100)$ $[\mathrm{M}]^{+}$. UV/Vis $\left(\mathrm{CH}_{2} \mathrm{Cl}_{2}\right): \lambda_{\max }(\varepsilon)=412 \mathrm{~nm}\left(69900 \mathrm{M}^{-1} \mathrm{~cm}^{-1}\right)$. Anal. Calcd for $\mathrm{C}_{56} \mathrm{H}_{74} \mathrm{O}_{13}(955.2)$ : C, 70.42; H, 7.81. Found: C, 69.97; H, 7.60.

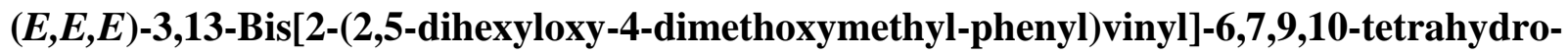
5,8,11-trioxa-dibenzo[a,e $]$ cyclotridecene (23a). Method a), yield: $1160 \mathrm{mg}$ (56\%) yellow crystals, mp 93-96 ${ }^{\circ} \mathrm{C} .{ }^{1} \mathrm{H}$ NMR $\left(\mathrm{CDCl}_{3}\right): \delta$ 0.87-0.92 (m, $\left.12 \mathrm{H}, \mathrm{CH}_{3}\right), 1.25-1.38\left(\mathrm{~m}, 16 \mathrm{H}, \mathrm{CH}_{2}\right)$, 1.43-1.55 (m, 8H, $\left.\mathrm{CH}_{2}\right), 1.76-1.86\left(\mathrm{~m}, 8 \mathrm{H}, \mathrm{CH}_{2}\right), 3.39\left(\mathrm{~s}, 12 \mathrm{H}, \mathrm{OCH}_{3}\right), 3.93-3.97(\mathrm{~m}, 4 \mathrm{H}$, $\left.\mathrm{OCH}_{2}\right), 3.96-4.04\left(\mathrm{~m}, 8 \mathrm{H}, \mathrm{OCH}_{2}\right), 4.19-4.23\left(\mathrm{~m}, 4 \mathrm{H}, \mathrm{OCH}_{2}\right), 5.60\left(\mathrm{~s}, 2 \mathrm{H}, \mathrm{CH}\left(\mathrm{OCH}_{3}\right)_{2}\right)$, 
7.05/7.43 (AB, ${ }^{3} J$ 16.2 Hz, 4H, olefin. H), 7.07 (s, 4H, aromat. H), 7.13 (s, 2H, H-4, H-12), 7.19 (d, $\left.{ }^{3} \mathrm{~J} 8.2 \mathrm{~Hz}, 2 \mathrm{H}, \mathrm{H}-2, \mathrm{H}-14\right), 7.47$ (d, $\left.{ }^{3} \mathrm{~J} 8.2 \mathrm{~Hz}, 2 \mathrm{H}, \mathrm{H}-1, \mathrm{H}-15\right), 7.84$ (s, 2H, H-16, H-17). ${ }^{13} \mathrm{C}$ NMR $\left(\mathrm{CDCl}_{3}\right): \delta 14.0\left(\mathrm{CH}_{3}\right), 22.6,25.8,29.4,31.6\left(\mathrm{CH}_{2}\right), 54.2\left(\mathrm{OCH}_{3}\right), 69.4,69.5,70.3,70.9$ $\left.\left(\mathrm{OCH}_{2}\right), 99.7\left(\mathrm{CH}_{(\mathrm{OCH}}\right)_{2}\right), 110.4,111.9,115.1,121.3,123.7,126.3,128.1,128.8$ (aromat. and olefin. $\mathrm{CH}), 127.3,127.4,128.9,138.0,150.9,150.9,157.6$ (aromat. $\left.\mathrm{C}_{\mathrm{q}}\right) . \mathrm{MS}(\mathrm{FD}), \mathrm{m} / \mathrm{z}(\%)=$ $1035(100)[\mathrm{M}]^{+}$. UV/Vis $\left(\mathrm{CH}_{2} \mathrm{Cl}_{2}\right): \lambda_{\max }(\varepsilon)=411 \mathrm{~nm}\left(67100 \mathrm{M}^{-1} \mathrm{~cm}^{-1}\right)$. Anal. Calcd for $\mathrm{C}_{64} \mathrm{H}_{90} \mathrm{O}_{11}$ (1035.4): C, 74.24; H, 8.76. Found: C, 73.94; H, 8.99.

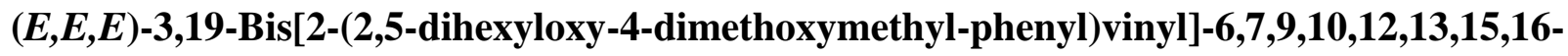
octahydro-5,8,11,14,17-pentaoxa-dibenzo[a,e]cyclononadecene (23b). Method a), yield: 1416 mg $(63 \%)$ yellow crystals, mp $128{ }^{\circ} \mathrm{C} .{ }^{1} \mathrm{H}$ NMR $\left(\mathrm{CDCl}_{3}\right): \delta \quad 0.87-0.94\left(\mathrm{~m}, 12 \mathrm{H}, \mathrm{CH}_{3}\right), 1.31-1.39$ $\left(\mathrm{m}, 16 \mathrm{H}, \mathrm{CH}_{2}\right), 1.43-1.54\left(\mathrm{~m}, 8 \mathrm{H}, \mathrm{CH}_{2}\right), 1.76-1.85\left(\mathrm{~m}, 8 \mathrm{H}, \mathrm{CH}_{2}\right), 3.39\left(\mathrm{~s}, 12 \mathrm{H}, \mathrm{OCH}_{3}\right), 3.69-3.77$ $\left(\mathrm{m}, 8 \mathrm{H}, \mathrm{OCH}_{2}\right), 3.97-4.06\left(\mathrm{~m}, 8 \mathrm{H}, \mathrm{OCH}_{2}\right), 4.22-4.27\left(\mathrm{~m}, 4 \mathrm{H}, \mathrm{OCH}_{2}\right), 5.61\left(\mathrm{~s}, 2 \mathrm{H}, \mathrm{CH}\left(\mathrm{OCH}_{3}\right)_{2}\right)$, 7.02 (s, 2H, H-4, H-18), 7.07, 7.08 (2s, 4H, aromat. H), 7.07/7.43 (AB, ${ }^{3} J$ 16.1 Hz, 4H, olefin. H), 7.13 (d, $\left.{ }^{3} J 8.0 \mathrm{~Hz}, 2 \mathrm{H}, \mathrm{H}-2, \mathrm{H}-20\right), 7.41$ (d, $\left.{ }^{3} \mathrm{~J} 8.0 \mathrm{~Hz}, 2 \mathrm{H}, \mathrm{H}-1, \mathrm{H}-21\right), 7.45$ (s, 2H, H-22, H23). ${ }^{13} \mathrm{C} \mathrm{NMR}\left(\mathrm{CDCl}_{3}\right): \delta 14.0\left(\mathrm{CH}_{3}\right), 22.6,25.8,25.9,29.5,31.6\left(\mathrm{CH}_{2}\right.$, partly superimposed), $54.2\left(\mathrm{OCH}_{3}\right), 68.0,69.4,69.6,70.0,71.3,71.7\left(\mathrm{OCH}_{2}\right), 99.7\left(\mathrm{CH}\left(\mathrm{OCH}_{3}\right)_{2}\right), 110.3,110.5,112.0$, 119.6, 125.7, 126.9, 129.0, 129.1 (aromat. and olefin. CH), 127.2, 127.3, 127.5, 138.1, 150.9, 151.0, 156.9 (aromat. $\mathrm{C}_{\mathrm{q}}$ ). MS (FD), $m / z(\%)=1123(100)[\mathrm{M}]^{+}$. UV/Vis $\left(\mathrm{CH}_{2} \mathrm{Cl}_{2}\right): \lambda_{\max }(\varepsilon)=$ $411 \mathrm{~nm}\left(62000 \mathrm{M}^{-1} \mathrm{~cm}^{-1}\right)$. Anal. Calcd for $\mathrm{C}_{68} \mathrm{H}_{98} \mathrm{O}_{13}$ (1123.5): C, 72.70; H, 8.79. Found: C, 72.33; H, 8.39.

Preparation of the Dialdehydes 25a,b-27a,b. The acetals 21a,b-23a,b (0.2 mmol), dissolved in $50 \mathrm{~mL}$ of $\mathrm{CHCl}_{3}$, were vigorously stirred with $30 \mathrm{~mL}$ of $10 \% \mathrm{HCl}$ for $2 \mathrm{~h}$. The organic phase was dried $\left(\mathrm{Na}_{2} \mathrm{SO}_{4}\right)$ and evaporated. A saturated solution of the residue in $\mathrm{CH}_{2} \mathrm{Cl}_{2}$ was then slowly dropped into a mixture of petroleum ether (bp 40-70 ${ }^{\circ} \mathrm{C}$ ) and diethylether (10:1) or into $n$ hexane. The formed precipitate is analytically pure.

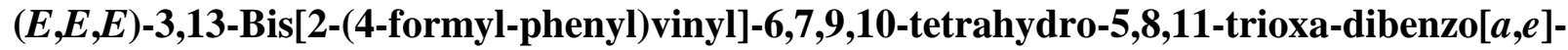
cyclotridecene (25a). Yellow-orange powder; yield: $93 \mathrm{~g}(86 \%)$; mp > $300{ }^{\circ} \mathrm{C} .{ }^{1} \mathrm{H} \mathrm{NMR}$ $\left(\mathrm{CDCl}_{3}\right): \delta 3.94-4.02\left(\mathrm{~m}, 4 \mathrm{H}, \mathrm{OCH}_{2}\right), 4.20-4.28\left(\mathrm{~m}, 4 \mathrm{H}, \mathrm{OCH}_{2}\right), 7.12 / 7.20\left(\mathrm{AB},{ }^{3} J 16.2 \mathrm{~Hz}, 4 \mathrm{H}\right.$, olefin. H), 7.16 (s, 2H, H-4, H-12), 7.21 (d, $\left.{ }^{3} J 8.2 \mathrm{~Hz}, 2 \mathrm{H}, \mathrm{H}-14\right), 7.50$ (d, ${ }^{3} J 8.2 \mathrm{~Hz}, \mathrm{H}-2, \mathrm{H}-1$, $\mathrm{H}-15$ ), 7.64/7.86 (AA'BB', 8H, aromat. H), 7.87 (s, 2H, olefin. H), 9.95 (s, 2H, CHO). Owing to the very low solubility, a ${ }^{13} \mathrm{C}$ NMR spectrum was not measured. MS (FD), $m / z(\%)=542(100)$ $[\mathrm{M}]^{+}$. UV/Vis $\left(\mathrm{CH}_{2} \mathrm{Cl}_{2}\right): \lambda_{\max }(\varepsilon)=421 \mathrm{~nm}\left(69300 \mathrm{M}^{-1} \mathrm{~cm}^{-1}\right)$. Anal. Calcd for $\mathrm{C}_{36} \mathrm{H}_{30} \mathrm{O}_{5}(542.6)$ : C, 79.69; H, 5.57. Found: C, 79.31; H, 5.17.

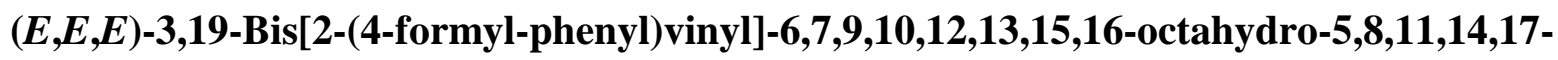
pentaoxa-dibenzo[a,e $]$ cyclononadecene (25b). Yellow-orange powder; yield: $109 \mathrm{mg}(86 \%)$; mp $219{ }^{\circ} \mathrm{C} .{ }^{1} \mathrm{H}$ NMR $\left(\mathrm{CD}_{2} \mathrm{Cl}_{2}\right): \delta$ 3.68-3.72 (m, 4H, $\left.\mathrm{OCH}_{2}\right), 3.72-3.76\left(\mathrm{~m}, 4 \mathrm{H}, \mathrm{OCH}_{2}\right), 4.03-$ $4.07\left(\mathrm{~m}, 4 \mathrm{H}, \mathrm{OCH}_{2}\right), 4.25-4.29\left(\mathrm{~m}, 4 \mathrm{H}, \mathrm{OCH}_{2}\right), 7.12$ (s, 2H, H-4, H-18), 7.19/7.28 (AB, ${ }^{3} J 16.4$ 
Hz, 4H, olefin. H), 7.20 (d, $\left.{ }^{3} J 8.1 \mathrm{~Hz}, 2 \mathrm{H}, \mathrm{H}-2, \mathrm{H}-20\right), 7.48$ (d, ${ }^{3} J$ 8.1 Hz, 2H, H-1, H-21), 7.49 (s, 2H, H-22, H-23), 7.69/7.87 (AA'BB', 8H, aromat. H), 9.98 (s, 2H, CHO). ${ }^{13} \mathrm{C}$ NMR $\left(\mathrm{CD}_{2} \mathrm{Cl}_{2}\right): \delta \quad 68.6,70.3,71.6,72.2\left(\mathrm{OCH}_{2}\right), 110.8,120.5,127.4,127.6,127.8,129.6,130.6$, 132.3 (aromat. and olefin. $\mathrm{CH}$ ), 128.5, 136.1, 137.4, 143.9, 157.5 (aromat. $\mathrm{C}_{\mathrm{q}}$ ), 191.8 (CHO). MS (FD), $m / z(\%)=630(100)[\mathrm{M}]^{+}$. UV/Vis $\left(\mathrm{CH}_{2} \mathrm{Cl}_{2}\right): \lambda_{\max }(\varepsilon)=421 \mathrm{~nm}\left(55000 \mathrm{M}^{-1} \mathrm{~cm}^{-1}\right)$. Anal. Calcd for $\mathrm{C}_{40} \mathrm{H}_{38} \mathrm{O}_{7}$ (630.5): C, 76.17; H, 6.07. Found: C, 76.53; H, 5.71.

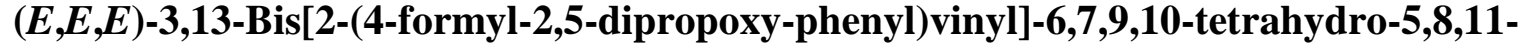

trioxa-dibenzo[a,e $]$ cyclotridecene (26a). Orange crystals; yield: $129 \mathrm{~g}(83 \%)$; mp 168-176 ${ }^{\circ} \mathrm{C}$. ${ }^{1} \mathrm{H} \mathrm{NMR}\left(\mathrm{CDCl}_{3}\right): \delta 1.07\left(\mathrm{t},{ }^{3} \mathrm{~J}=7.2 \mathrm{~Hz}, 12 \mathrm{H}, \mathrm{CH}_{3}\right), 1.82-1.92\left(\mathrm{~m}, 8 \mathrm{H}, \mathrm{CH}_{2}\right), 3.93-3.96(\mathrm{~m}, 4 \mathrm{H}$, $\left.\mathrm{OCH}_{2}\right), 3.96\left(\mathrm{t},{ }^{3} J 7.3 \mathrm{~Hz}, 4 \mathrm{H}, \mathrm{OCH}_{2}\right), 4.06\left(\mathrm{t},{ }^{3} J\right.$ 7.3 Hz, 4H, $\left.\mathrm{OCH}_{2}\right), 4.20-4.24\left(\mathrm{~m}, 4 \mathrm{H}, \mathrm{OCH}_{2}\right)$, 7.14-7.17 (m, 4H, H-4, H-12, aromat. H), 7.19/7.45 (AB, ${ }^{3} J 16.4 \mathrm{~Hz}, 4 \mathrm{H}$, olefin. H), 7.22 (d, ${ }^{3} J$ $8.2 \mathrm{~Hz}, 2 \mathrm{H}, \mathrm{H}-2, \mathrm{H}-14), 7.31$ (s, 2H, aromat. H), 7.50 (d, $\left.{ }^{3} \mathrm{~J} 8.2 \mathrm{~Hz}, 2 \mathrm{H}, \mathrm{H}-1, \mathrm{H}-15\right), 7.86$ (s, 2H, olefin. H), 10.44 (s, 2H, CHO). ${ }^{13} \mathrm{C} \mathrm{NMR}\left(\mathrm{CDCl}_{3}\right): \delta 10.6\left(\mathrm{CH}_{3}\right), 22.6,22.7\left(\mathrm{CH}_{2}\right), 70.3,70.8$, 70.9, $71.0\left(\mathrm{OCH}_{2}\right), 110.5,110.8,115.5,121.7,123.1,126.6,128.3,131.9$ (aromat. and olefin. $\mathrm{CH}), 124.5,129.6,134.3,137.4,150.9,156.3,157.7$ (aromat. $\mathrm{C}_{\mathrm{q}}$ ), 189.0 (CHO). MS (FD), $\mathrm{m} / z$ $(\%)=774(100)[\mathrm{M}]^{+}$. UV/Vis $\left(\mathrm{CH}_{2} \mathrm{Cl}_{2}\right): \lambda_{\max }(\varepsilon)=438 \mathrm{~nm}\left(58100 \mathrm{M}^{-1} \mathrm{~cm}^{-1}\right)$. Anal. Calcd for $\mathrm{C}_{48} \mathrm{H}_{54} \mathrm{O}_{9}$ (775.0): C, 74.40; H, 7.02. Found: C, 74.02; H, 6.73.

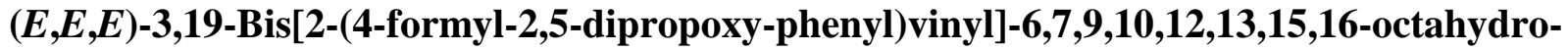

5,8,11,14,17-pentaoxa-dibenzo[a,e]cyclononadecene (26b). Orange powder; yield: $154 \mathrm{mg}$ (89\%); mp 92-102 ${ }^{\circ} \mathrm{C} .{ }^{1} \mathrm{H}$ NMR $\left(\mathrm{CDCl}_{3}\right): \delta 1.08\left(\mathrm{t},{ }^{3} \mathrm{~J} 7.2 \mathrm{~Hz}, 12 \mathrm{H}, \mathrm{CH}_{3}\right), 1.75-1.95(\mathrm{~m}, 8 \mathrm{H}$, $\left.\mathrm{CH}_{2}\right), 3.65-3.78\left(\mathrm{~m}, 8 \mathrm{H}, \mathrm{OCH}_{2}\right), 3.93-4.10\left(\mathrm{~m}, 12 \mathrm{H}, \mathrm{OCH}_{2}\right), 4.20-4.22\left(\mathrm{~m}, 4 \mathrm{H}, \mathrm{OCH}_{2}\right), 7.05$ (s, 2H, H-4, H-18), 7.12-7.19 (m, 4H, H-2, H-20, aromat. H), 7.21/7.45 (AB, ${ }^{3} J$ 16.2 Hz, 4H, olefin. H), 7.31 (s, 2H, aromat. H), 7.39-7.51 (m, 2H, H-1, H-21), 7.47 (s, 2H, H-22, H-23). ${ }^{13} \mathrm{C}$ $\operatorname{NMR}\left(\mathrm{CDCl}_{3}\right): \delta 10.6,10.5\left(\mathrm{CH}_{3}\right), 22.6\left(\mathrm{CH}_{2}\right), 68.0,70.0,70.7,70.9,71.2,71.7\left(\mathrm{OCH}_{2}\right), 110.5$, $110.5,110.8,119.9,122.9,127.2,127.9,129.1$ (aromat. and olefin. $\mathrm{CH}$ ), 124.5, 132.1, 134.3,

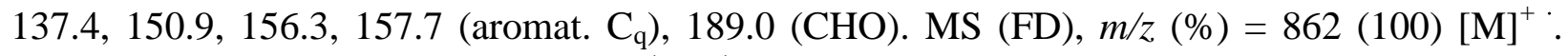
UV/Vis: $\lambda_{\max }(\varepsilon)=438 \mathrm{~nm}\left(70000 \mathrm{M}^{-1} \mathrm{~cm}^{-1}\right)$. Anal. Calcd for $\mathrm{C}_{52} \mathrm{H}_{62} \mathrm{O}_{11}$ (863.1): C, 72.37; $\mathrm{H}$, 7.24. Found: C, 72.54; H, 7.32.

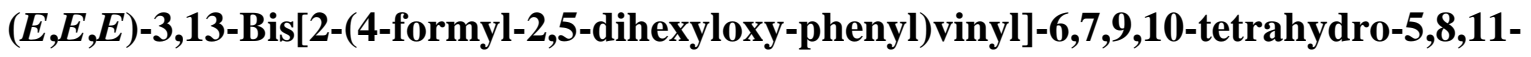
trioxa-dibenzo[a,e $]$ cyclotridecene (27a). Orange crystals; yield: $170 \mathrm{mg}(90 \%) ; \mathrm{mp} 99{ }^{\circ} \mathrm{C} .{ }^{1} \mathrm{H}$ NMR $\left(\mathrm{CDCl}_{3}\right): \delta$ 0.88-0.95 (m, 12H, $\left.\mathrm{CH}_{3}\right), 130-1.40\left(\mathrm{~m}, 16 \mathrm{H}, \mathrm{CH}_{2}\right), 1.45-1.55\left(\mathrm{~m}, 8 \mathrm{H}, \mathrm{CH}_{2}\right)$, 1.80-1.88 (m, 8H, $\left.\mathrm{CH}_{2}\right), 3.93-3.98\left(\mathrm{~m}, 4 \mathrm{H}, \mathrm{OCH}_{2}\right), 3.98-4.04\left(\mathrm{~m}, 4 \mathrm{H}, \mathrm{OCH}_{2}\right), 4.06-4.12(\mathrm{~m}, 4 \mathrm{H}$, $\left.\mathrm{OCH}_{2}\right), 4.20-4.25\left(\mathrm{~m}, 4 \mathrm{H}, \mathrm{CH}_{2}\right), 7.14-7.18$ (m, 4H, H-4, H-12, aromat. H), 7.19/7.44 (AB, ${ }^{3} J$ $16.4 \mathrm{~Hz}, 4 \mathrm{H}$, olefin. H), 7.20-7.25 (m, 2H, H-2, H-14), 7.31 (s, 2H, aromat. H), $7.50\left({ }^{3} \mathrm{~J} 7.9 \mathrm{~Hz}\right.$, 2H, H-1, H-15), 7.87 (s, 2H, olefin. H), 10.43 (s, 2H, CHO). ${ }^{13} \mathrm{C} \mathrm{NMR}\left(\mathrm{CDCl}_{3}\right): \delta 14.0\left(\mathrm{CH}_{3}\right)$, 22.6, 22.6, 25.8, 25.9, 29.2, 29.2, 31.5, $31.6\left(\mathrm{CH}_{2}\right), 69.3,69.4,70.3,70.9\left(\mathrm{OCH}_{2}\right), 110.4,110.8$, $115.4,121.7,123.1,126.6,128.3,131.8$ (aromat. and olefin. $\mathrm{CH}$ ), 124.5, 129.6, 134.3, 137.4, 
150.9, 156.3, 157.7 (aromat. $\mathrm{C}_{\mathrm{q}}$ ), $189.0(\mathrm{CHO})$. MS (FD), $\mathrm{m} / \mathrm{z}(\%)=943(100)[\mathrm{M}]^{+}$. UV/Vis: $\lambda_{\max }(\varepsilon)=438 \mathrm{~nm}\left(66400 \mathrm{M}^{-1} \mathrm{~cm}^{-1}\right)$. Anal. Calcd for $\mathrm{C}_{60} \mathrm{H}_{78} \mathrm{O}_{9}(943.7): \mathrm{C}, 76.40 ; \mathrm{H}, 8.33$. Found: C, $76.11 ; \mathrm{H}, 8.51$.

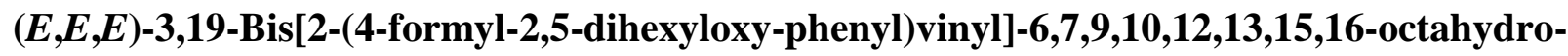
$\mathbf{5 , 8 , 1 1 , 1 4 , 1 7}$-pentaoxa-dibenzo[a,e]cyclononadecene (27b). Orange powder, yield: $192 \mathrm{mg}$ (93\%), mp $95{ }^{\circ} \mathrm{C} .{ }^{1} \mathrm{H}$ NMR $\left(\mathrm{CDCl}_{3}\right): \delta \quad 0.81-0.95\left(\mathrm{~m}, 12 \mathrm{H}, \mathrm{CH}_{3}\right), 125-1.40\left(\mathrm{~m}, 16 \mathrm{H}, \mathrm{CH}_{2}\right), 1.40-$ $1.60\left(\mathrm{~m}, 8 \mathrm{H}, \mathrm{CH}_{2}\right), 1.75-1.95\left(\mathrm{~m}, 8 \mathrm{H}, \mathrm{CH}_{2}\right), 3.66-3.77\left(\mathrm{~m}, 8 \mathrm{H}, \mathrm{OCH}_{2}\right), 3.95-4.15(\mathrm{~m}, 12 \mathrm{H}$, $\left.\mathrm{OCH}_{2}\right), 4.20-4.30\left(\mathrm{~m}, 4 \mathrm{H}, \mathrm{OCH}_{2}\right), 7.05$ (s, 2H, H-4, H-18), 7.12-7.19 (m, 4H, H-2, H-20, aromat. $\mathrm{H}), 7.21 / 7.44\left(\mathrm{AB},{ }^{3} J 16.2 \mathrm{~Hz}, 4 \mathrm{H}\right.$, olefin. $\left.\mathrm{H}\right), 7.31$ (s, 2H, aromat. H), 7.39-7.50 (m, 4H, H-1, $\mathrm{H}-21, \mathrm{H}-22, \mathrm{H}-23), 10.43$ (s, 2H, CHO). ${ }^{13} \mathrm{C} \mathrm{NMR}\left(\mathrm{CDCl}_{3}\right): \delta 13.9,14.0\left(\mathrm{CH}_{3}\right), 22.5,22.6,25.7$, 25.9, 29.2, 29.2, 31.5, $31.5\left(\mathrm{CH}_{2}\right), 68.0,69.2,69.4,69.9,71.2,71.7\left(\mathrm{OCH}_{2}\right), 110.4,110.5,110.8$, 119.9, 123.0, 127.2, 129.1, 132.1 (aromat. and olefin. $\mathrm{CH}$ ), 124.4, 127.9, 134.3, 137.4, 150.8, 156.2, 156.9 (aromat. $\mathrm{C}_{\mathrm{q}}$ ), $189.0(\mathrm{CHO})$. MS (FD), $m / z(\%)=1031(100)[\mathrm{M}]^{+\cdot}$. UV/Vis: $\lambda_{\max }(\varepsilon)$ $=438 \mathrm{~nm}\left(73900 \mathrm{M}^{-1} \mathrm{~cm}^{-1}\right)$. Anal. Calcd for $\mathrm{C}_{64} \mathrm{H}_{86} \mathrm{O}_{11}(1031.4): \mathrm{C}, 74.53 ; \mathrm{H}, 8.40$. Found: $\mathrm{C}$, 74.23; H, 8.11.

1,4-Phenylenevinylene tetramers with two stilbene crowns 30a,b and 31a,b. Dialdehyde 9a or $\mathbf{9 b}(0.6 \mathrm{mmol})$, diphosphonate $\mathbf{2 8}$ or $\mathbf{2 9},(10.2 \mathrm{mmol}), 85 \% \mathrm{KOH}(50 \mathrm{~m}, 0.76 \mathrm{mmol}), 0.50 \mathrm{~g}$ Aliquat $336,0.5 \mathrm{~mL}$ of $\mathrm{H}_{2} \mathrm{O}$ and $100 \mathrm{~mL}$ of benzene were heated to reflux for $24 \mathrm{~h} . \mathrm{H}_{2} \mathrm{O}(10$ $\mathrm{mL}$ ) was added and the organic phase separated and evaporated. The residue was treated with 5$10 \mathrm{~mL}$ of $\mathrm{CH}_{2} \mathrm{Cl}_{2}$. The filtered solution was slowly dropped in petroleum ether (bp $40-70{ }^{\circ} \mathrm{C}$ ), where the product as red solid precipitated. (The major, insoluble part in $\mathrm{CH}_{2} \mathrm{Cl}_{2}$ contained higher oligomers).

(E,E,E,E)-PV-Tetramer 30a. Yield: $21 \mathrm{mg}(11 \%)$ red solid, mp 275-280 ${ }^{\circ} \mathrm{C} .{ }^{1} \mathrm{H}$ NMR $\left(\mathrm{CD}_{2} \mathrm{Cl}_{2}\right): \delta 1.14\left(\mathrm{t},{ }^{3} J=7.6 \mathrm{~Hz}, 6 \mathrm{H}, \mathrm{CH}_{3}\right), 1.87-1.95\left(\mathrm{~m}, 4 \mathrm{H}, \mathrm{CH}_{2}\right), 3.92-4.10\left(\mathrm{~m}, 12 \mathrm{H}, \mathrm{OCH}_{2}\right)$, 4.18-4.29 (m, 8H, $\left.\mathrm{OCH}_{2}\right), 7.13-7.25(\mathrm{~m}, 6 \mathrm{H}$, aromat. and olefin. $\mathrm{H}), 7.25-7.33\left(\mathrm{~d},{ }^{3} J \quad 8.2 \mathrm{~Hz}, 2 \mathrm{H}\right.$, aromat. H), 7.49 (s, 2H, aromat. H), $7.53\left(\mathrm{~d},{ }^{3} J 16.2 \mathrm{~Hz}, 2 \mathrm{H}\right.$, olefin. H), 7.54 (d, ${ }^{3} J 7.9 \mathrm{~Hz}, 2 \mathrm{H}$, aromat. H), $7.57\left(\mathrm{~d},{ }^{3} J 7.9 \mathrm{~Hz}, 2 \mathrm{H}\right.$, aromat. $\left.\mathrm{H}\right), 7.65\left(\mathrm{~d},{ }^{3} J 7.9 \mathrm{~Hz}, 2 \mathrm{H}\right.$, aromat. $\left.\mathrm{H}\right), 7.79\left(\mathrm{~d},{ }^{3} J\right.$ $16.7 \mathrm{~Hz}, 2 \mathrm{H}$, olefin. H), $8.13\left(\mathrm{~d},{ }^{3} \mathrm{~J} 16.7 \mathrm{~Hz}, 2 \mathrm{H}\right.$, olefin. $\mathrm{H}$ ), 9.93 (s, $\left.2 \mathrm{H}, \mathrm{CHO}\right) .{ }^{13} \mathrm{C} \mathrm{NMR}$ $\left(\mathrm{CDCl}_{3}\right): \delta 11.0\left(\mathrm{CH}_{3}\right), 23.3\left(\mathrm{CH}_{2}\right), 70.4,70.7,71.1,71.6,71.8\left(\mathrm{OCH}_{2}\right), 111.1,116.0,116.3$, 121.8, 124.4, 125.3, 125.5, 128.5, 128.7, 129.0, 130.4 (aromat. and olefin. $\mathrm{CH}$ ), 127.4, 128.7, 136.0, 136.4, 139.5, 151.7, 158.1, 158.5 (aromat. $\mathrm{C}_{\mathrm{q}}$ ), $191.5(\mathrm{CHO})$. MS (FD), $\mathrm{m} / z(\%)=946$ $(100)[\mathrm{M}]^{+}$. UV/Vis $\left(\mathrm{CH}_{2} \mathrm{Cl}_{2}\right): \lambda_{\max }(\varepsilon)=447 \mathrm{~nm}\left(104300 \mathrm{M}^{-1} \mathrm{~cm}^{-1}\right)$. Anal. Calcd for $\mathrm{C}_{60} \mathrm{H}_{66} \mathrm{O}_{10}$ (946.6): C, 76.09; H, 7.02. Found: C, 75.73; H, 7.37.

(E,E,E,E)-PV-Tetramer 30b. Yield: $72 \mathrm{mg}(35 \%)$, red solid, mp $234{ }^{\circ} \mathrm{C} .{ }^{1} \mathrm{H} \mathrm{NMR}\left(\mathrm{CDCl}_{3}\right): \delta$ $1.12\left(\mathrm{t},{ }^{3} \mathrm{~J}=7.6 \mathrm{~Hz}, 6 \mathrm{H}, \mathrm{CH}_{3}\right), 1.82-2.00\left(\mathrm{~m}, 4 \mathrm{H}, \mathrm{CH}_{2}\right), 3.63-3.79\left(\mathrm{~m}, 16 \mathrm{H}, \mathrm{OCH}_{2}\right), 3.95-4.10$ $\left(\mathrm{m}, 12 \mathrm{H}, \mathrm{OCH}_{2}\right), 4.20-4.32\left(\mathrm{~m}, 8 \mathrm{H}, \mathrm{OCH}_{2}\right), 7.05(\mathrm{~s}, 2 \mathrm{H}$, aromat. $\mathrm{H}), 7.08-7.18(\mathrm{~m}, 6 \mathrm{H}$, aromat. 
and olefin. H), 7.37-7.52 (m, 10H, aromat. and olefin. $\mathrm{H})$, 7.54-7.64 (m, 4H, aromat. and olefin. $\mathrm{H}), 9.93(\mathrm{~s}, 2 \mathrm{H}, \mathrm{CHO}) .{ }^{13} \mathrm{C} \mathrm{NMR}\left(\mathrm{CDCl}_{3}\right): \delta 10.7\left(\mathrm{CH}_{3}\right), 22.9\left(\mathrm{CH}_{2}\right), 67.9,68.1,69.7,69.8$, 71.1, 71.2, 71.2, 71.5, $71.7\left(\mathrm{OCH}_{2}\right), 110.2,110.6,111.0,119.5,124.0,124.3,125.9,128.4$, 128.6, 129.9, 130.5 (aromat. and olefin. CH), 126.3, 127.0, 134.6, 136.0, 139.0, 151.3, 156.9, 157.2 (aromat. $\mathrm{C}_{\mathrm{q}}$ ), $191.4(\mathrm{CHO})$. MS (FD), $m / z(\%)=1039(100)[\mathrm{M}]^{+}$. UV/Vis $\left(\mathrm{CH}_{2} \mathrm{Cl}_{2}\right): \lambda_{\max }$ $(\varepsilon)=443 \mathrm{~nm}\left(111500 \mathrm{M}^{-1} \mathrm{~cm}^{-1}\right)$. Anal. Calcd for $\mathrm{C}_{62} \mathrm{H}_{70} \mathrm{O}_{14}$ (1039.2): C, 71.66; H, 6.79. Found: C, 71.42; H, 6.59 .

$(\boldsymbol{E}, \boldsymbol{E}, \boldsymbol{E}, \boldsymbol{E})$-PV-Tetramer 31a. Yield: $32 \mathrm{mg}(17 \%)$, red powder, mp $230{ }^{\circ} \mathrm{C} .{ }^{1} \mathrm{H} \mathrm{NMR}\left(\mathrm{CDCl}_{3}\right): \delta$ 0.89-0.95 (m, 6H, $\left.\mathrm{CH}_{3}\right), 1.33-1.43\left(\mathrm{~m}, 8 \mathrm{H}, \mathrm{CH}_{2}\right), 1.52-1.60\left(\mathrm{~m}, 4 \mathrm{H}, \mathrm{CH}_{2}\right), 1.83-1.92(\mathrm{~m}, 4 \mathrm{H}$, $\left.\mathrm{CH}_{2}\right), 3.92-4.00\left(\mathrm{~m}, 8 \mathrm{H}, \mathrm{OCH}_{2}\right), 4.05\left(\mathrm{t},{ }^{3} \mathrm{~J} 6.4 \mathrm{~Hz}, 4 \mathrm{H}, \mathrm{OCH}_{2}\right), 4.20-4.27\left(\mathrm{~m}, 8 \mathrm{H}, \mathrm{OCH}_{2}\right)$, 7.10/7.48 (AB, ${ }^{3} J$ 16.4 Hz, 4H, olefin. H), 7.10 (s, 2H, aromat. H), 7.18 (s, 2H, aromat. H), 7.237.27 (m, 2H, aromat. H), 7.46 (s, 2H, aromat. H), 7.50 (d, ${ }^{3} J 8.8 \mathrm{~Hz}, 2 \mathrm{H}$, aromat. H), 7.53 (d, ${ }^{3} J$ $8.2 \mathrm{~Hz}, 2 \mathrm{H}$, aromat. H), $7.60\left(\mathrm{~d},{ }^{3} J 7.9 \mathrm{~Hz}, 2 \mathrm{H}\right.$, aromat. $\left.\mathrm{H}\right), 7.72 / 8.16\left(\mathrm{AB},{ }^{3} J 16.7 \mathrm{~Hz}, 4 \mathrm{H}\right.$, olefin. H), 9.94 (s, 2H, CHO). ${ }^{13} \mathrm{C}$ NMR $\left(\mathrm{CDCl}_{3}\right): \delta 14.0\left(\mathrm{CH}_{3}\right), 22.6,26.0,29.6,31.6\left(\mathrm{CH}_{2}\right)$, 69.6, 70.1, 70.3, 70.4, $71.4\left(\mathrm{OCH}_{2}\right), 110.9,115.5,115.7,121.5,124.1,125.0,125.2,128.0$, 128.3, 128.7, 130.2 (aromat. and olefin. CH), 127.0, 135.7, 135.7, 139.1, 151.3, 157.6, 157.9 (aromat. $\mathrm{C}_{\mathrm{q}}$, partly superimposed), $191.2(\mathrm{CHO})$. MS (FD), $m / z(\%)=946(100)[\mathrm{M}]^{+}$. UV/Vis $\left(\mathrm{CH}_{2} \mathrm{Cl}_{2}\right): \lambda_{\max }(\varepsilon)=447 \mathrm{~nm}\left(100900 \mathrm{M}^{-1} \mathrm{~cm}^{-1}\right)$. Anal. Calcd for $\mathrm{C}_{60} \mathrm{H}_{66} \mathrm{O}_{10}$ (947.2): C, 76.09; $\mathrm{H}$, 7.02. Found: C, 75.89; H, 7.30.

$(\boldsymbol{E}, \boldsymbol{E}, \boldsymbol{E}, \boldsymbol{E})$-PV-Tetramer 31b. Yield: $25 \mathrm{mg}(11 \%)$, red crystals, mp $196{ }^{\circ} \mathrm{C} .{ }^{1} \mathrm{H} \mathrm{NMR}\left(\mathrm{CDCl}_{3}\right)$ : $\delta$ 0.89-0.95 (m, 6H, $\left.\mathrm{CH}_{3}\right), 1.33-1.43\left(\mathrm{~m}, 8 \mathrm{H}, \mathrm{CH}_{2}\right), 1.52-1.60\left(\mathrm{~m}, 4 \mathrm{H}, \mathrm{CH}_{2}\right), 1.83-1.92(\mathrm{~m}, 4 \mathrm{H}$, $\left.\mathrm{CH}_{2}\right), 3.68-3.77\left(\mathrm{~m}, 16 \mathrm{H}, \mathrm{OCH}_{2}\right), 4.00-4.04\left(\mathrm{t},{ }^{3} \mathrm{~J}=5.0 \mathrm{~Hz}, 4 \mathrm{H}, \mathrm{OCH}_{2}\right), 4.04-4.09(\mathrm{~m}, 8 \mathrm{H}$, $\left.\mathrm{OCH}_{2}\right), 4.24-4.30\left(\mathrm{~m}, 8 \mathrm{H}, \mathrm{OCH}_{2}\right), 7.04\left(\mathrm{~s}, 2 \mathrm{H}\right.$, aromat. H), 7.11/7.43 (AB, ${ }^{3} J 16.4 \mathrm{~Hz}, 4 \mathrm{H}$, olefin. H), 7.11 (s, 2H, aromat. H), 7.15 (d, ${ }^{3} J$ 8.2 Hz, 2H, aromat. H), 7.38 (s, 2H, aromat. H), $7.40\left(\mathrm{~d},{ }^{3} \mathrm{~J} 8.2 \mathrm{~Hz}, 2 \mathrm{H}\right.$, aromat. H), 7.42-7.46 (m, 2H, aromat. H), 7.48/7.57 (AB, ${ }^{3} J 16.4 \mathrm{~Hz}$, 4H, olefin. H), 7.60 (d, ${ }^{3} J \quad 7.8 \mathrm{~Hz}, 2 \mathrm{H}$, aromat. H), 9.92 (s, 2H, CHO). ${ }^{13} \mathrm{C} \mathrm{NMR}\left(\mathrm{CDCl}_{3}\right): \delta$ $14.0\left(\mathrm{CH}_{3}\right), 22.6,26.0,29.5,31.6\left(\mathrm{CH}_{2}\right), 67.9,68.1,69.6,69.7,69.9,71.2,71.3,71.5,71.7$ $\left(\mathrm{OCH}_{2}\right), 110.2,110.6,110.9,119.5,124.1,124.3,125.9,128.4,128.6,129.9,130.5$ (aromat. and olefin. CH), 126.3, 127.0, 134.6, 136.0, 139.0, 151.3, 156.9, 157.2 (aromat. $\mathrm{C}_{\mathrm{q}}$ ), 191.4 (CHO). MS (FD), $m / z(\%)=1122(100)[\mathrm{M}]^{+}$. UV/Vis $\left(\mathrm{CH}_{2} \mathrm{Cl}_{2}\right): \lambda_{\max }(\varepsilon)=443 \mathrm{~nm}\left(108200 \mathrm{M}^{-1} \mathrm{~cm}^{-1}\right)$. Anal. Calcd for $\mathrm{C}_{68} \mathrm{H}_{82} \mathrm{O}_{14}$ (1123.3): C, 72.70; H, 7.36. Found: C, 72.70; H, 7.06 (incomplete combustion).

all-(E)-Nonamer 34. Dialdehyde 27a (114 mg, $0.12 \mathrm{mmol})$, diphosphonate 8a (35 mg, 0.06 $\mathrm{mmol}), \mathrm{KOH}(85 \%, 20 \mathrm{mg}, 0.42 \mathrm{mmol})$ and Aliquat $336(10 \mathrm{mg})$ were heated to reflux in 300 $\mathrm{mL}$ of benzene/ $0.5 \mathrm{~mL} \mathrm{H}_{2} \mathrm{O}$ for $3 \mathrm{~d}$. After the addition of $50 \mathrm{~mL}$ of $\mathrm{H}_{2} \mathrm{O}$, the mixture was stirred at r. t. and the precipitate filtered off, dried and extracted with hot $\mathrm{CHCl}_{3}$. The soluble part consisted of the red solid $34(16 \mathrm{mg}, 12 \%)$ which melted at $245{ }^{\circ} \mathrm{C} .{ }^{1} \mathrm{H} \mathrm{NMR}\left(\mathrm{CD}_{2} \mathrm{Cl}_{2}\right): \delta 0.89$ $0.95\left(\mathrm{~m}, 24 \mathrm{H}, \mathrm{CH}_{3}\right), 1.30-1.75\left(\mathrm{~m}, 48 \mathrm{H}, \mathrm{CH}_{2}\right), 1.80-1.90\left(\mathrm{~m}, 16 \mathrm{H}, \mathrm{CH}_{2}\right), 3.80-4.30(\mathrm{~m}, 40 \mathrm{H}$, 
$\mathrm{OCH}_{2}$ ), 7.00-7.60 (m, 38H, aromat. and olefin. $\left.\mathrm{H}\right), 7.80-7.90$ (m, $6 \mathrm{H}$, olefin. $\mathrm{H}$ in crown), 9.74 (s, 2H, CHO). MS (FD), $m / z(\%)=2160(20)[\mathrm{M}]^{+*}, 671(100)$. UV/Vis $\left(\mathrm{CH}_{2} \mathrm{Cl}_{2}\right): \lambda_{\max }(\varepsilon)=457$ $\mathrm{nm}$ (very low solubility, $\varepsilon$ not determined). Anal. Calcd for $\mathrm{C}_{140} \mathrm{H}_{174} \mathrm{O}_{19}$ (2160.9): C, 77.82; H, 8.12. Found: C, 77.54; $\mathrm{H}, 8.31$.

\section{Acknowledgements}

We are grateful to the Deutsche Forschungsgemeinschaft and the Fonds der Chemischen Industrie for financial support.

\section{References}

1. Goel, M.; Jayakannan, M. Chem. Eur. J. 2012, 18, 2867-2874. http://dx.doi.org/10.1002/chem.201102670, PMid:22298381

2. Bettini, S.; Valli, L.; Giancane, G.; Santino, A.; Martinelli, C.; Farinola, G. M.; Cardone, A.; Sgobba, V. Dyes and Pigments 2012, 94, 156-162. http://dx.doi.org/10.1016/j.dyepig.2011.12.010

3. Nemkovich, N. A.; Detert, H.; Schmitt, V. Chem. Phys. 2010, 378, 37-41. http://dx.doi.org/10.1016/j.chemphys.2010.09.011

4. Moorthy, J. N.; Venkatakrishnan, P.; Natarajan, P.; Lin, Z.; Chow, T. J. J. Org. Chem. 2010, 75, 2599-2609. http://dx.doi.org/10.1021/jo1001565, PMid:20329712

5. Goel, M. ; Jayakannan, M. J. Phys. Chem. B 2010, 114, 12508-12519. http://dx.doi.org/10.1021/jp105839f, PMid:20726547

6. Kim, S.; Kalbitz, H.; Hillmann, S.; Meier, H. Eur. J. Org. Chem. 2009, 1976-1983. http://dx.doi.org/10.1002/ejoc.200801165

7. Lifka, T.; Kalbitz, H.; Meier, H. Z. Naturforsch. 2009, 64b, 1183-1186.

8. Kim, S.; Oehlhof, A.; Beile, B.; Meier, H. Helv. Chim. Acta 2009, 92, 1023-1033. http://dx.doi.org/10.1002/hlca.200800398

9. Meier, H.; Lifka, T.; Stalmach, U.; Oehlhof, A.; Prehl, S. Eur. J. Org. Chem. 2008, 15681574. http://dx.doi.org/10.1002/ejoc.200701046

10. Amrutha, S. R.; Jayakannan, M. J. Phys. Chem. 2008, 112, 1119-1129.

11. Detert, H.; Lehmann, M.; Meier, H. Materials 2010, 3, 3218-3330. http://dx.doi.org/10.3390/ma3053218

12. Lin, H.-C.; Jin, B.-Y. Materials 2010, 3, 4214-4251. http://dx.doi.org/10.3390/ma3084214 
13. Li, C.; Liu, M.; Pschirer, N. G.; Baumgarten, M.; Müllen, K. Chem. Rev. 2010, 110, 68176855. http://dx.doi.org/10.1021/cr100052z, PMid:20583837

14. Babudri, F.; Farinola, G. M.; Naso, F. Synlett 2009, 17, 2740-2748.

15. Figueira-Duarte, T. M.; Gegout, A.; Nierengarten, J.-F. Chem. Commun. 2007, 2, 109-119. http://dx.doi.org/10.1039/b609383c, PMid:17180218

16. Meier, H. in Molecules to Materials; Haley, M. M.; Tykwinski, R. R. Eds.; Wiley-VCH: Weinheim, 2006; p 476.

17. Meier, H. Angew. Chem. 2005, 117, 2536-2561; Angew. Chem. Int. Ed. Engl. 2005, 44, 2482-2506. http://dx.doi.org/10.1002/anie.200461146, PMid:15846835

18. Meier, H.; Lehmann, M. In Encyclopedia of Nanoscience and Nanotechnology; Nalwa, H. S. Ed.; American Scientific Publishers: Stevenson Ranch, 2004; Vol. 10, pp 95-106.

19. Wong, M. S.; Li, Z. H. Pure Appl. Chem. 2004, 76, 1409-1419. http://dx.doi.org/10.1351/pac200476071409

20. Gierschner, J.; Oelkrug, D. In Encyclopedia of Nanoscience and Nanotechnology; Nalwa, H. S. Ed.; American Scientific Publishers: Stevenson Ranch, 2004; Vol. 8, p 219-238.

21. Segura, J. L.; Martin, N. J. Mater. Chem. 2000, 10, 2403-2435. http://dx.doi.org/10.1039/b004407p

22. Martin, R. E.; Diederich, F. Angew. Chem. 1999, 111, 1440-1469; Angew. Chem. Int. Ed. Engl. 1999, 38, 1350-1377. http://dx.doi.org/10.1002/(SICI)1521-3773(19990517)38:10<1350::AIDANIE1350>3.0.CO;2-6

23. Scherf, U. Top. Curr. Chem. 1999, 201, 163-222. http://dx.doi.org/10.1007/3-540-49451-0_5

24. Müllen, K.; Wegner, G. Electronic Materials: The Oligomer Approach; Wiley-VCH: Weinheim, 1998. http://dx.doi.org/10.1002/9783527603220

25. Kraft, A.; Grimsdale, A. C.; Holmes, A. B. Angew. Chem. 1998, 110, 416-443; Angew. Chem. Int. Ed. Engl. 1998, 37, 403-428.

26. Tirado-Rives, J.; Gandour, R. D.; Fronczek, F. R. Tetrahedron Lett. 1982, 23, 1639-1642. http://dx.doi.org/10.1016/S0040-4039(00)87178-6

27. Tirado-Rives, J.; Oliver, M. A.; Fronczek, F. R.; Gandour, R. D. J. Org. Chem. 1984, 49, 1627-1634. http://dx.doi.org/10.1021/jo00183a026

28. Merz, A.; Karl, A.; Futterer, T.; Stacherdinger, N.; Schneider, O.; Lex, J.; Luboch, E.; Biernat, J. F. Liebigs Ann. Chem. 1994, 1199-1210. http://dx.doi.org/10.1002/jlac.199419941211

29. Fürstner, A.; Seidel, G.; Kopiske, C.; Krüger, C.; Mynott, R. Liebigs Ann. Chem. 1996, 655662. 
30. Merz, A.; Futterer, T.; Lex, J.; Inerowicz, H. Angew. Chem. 1997, 109, 293-295; Angew. Chem. Int. Ed. Engl. 1997, 36, 278-280. http://dx.doi.org/10.1002/anie.199702781

31. Futterer, T.; Merz, A.; Lex, J. Angew. Chem. 1997, 109, 619-622; Angew. Chem. Int. Ed. Engl. 1997, 36, 611-613. http://dx.doi.org/10.1002/anie.199706111

32. Barbosa, F.; Peron, V.; Gescheidt, G.; Fürstner, A. J. Org. Chem. 1998, 63, 8806-8814. http://dx.doi.org/10.1021/jo980920o

33. Callan, J. F.; De Silva, A. P.; Magri, D. C. Tetrahedron 2005, 61, 8551-8588. http://dx.doi.org/10.1016/j.tet.2005.05.043

34. Phan, D. H. T.; Kim, B.; Dong, V. M. J. Am. Chem. Soc. 2009, 131, 15608-15609. http://dx.doi.org/10.1021/ja907711a, PMid:19813746

35. Jadhav, A. H.; Kim, H.; Wang, I. T. Catalysis Communications 2012, 21, 96-103. http://dx.doi.org/10.1016/j.catcom.2012.02.007

36. Ingham, A. M.; Xu, C.; Whitcombe, T. W.; Bridson, J. N.; McAuley, A. Canad. J. Chem. 2002, 80, 155-162. http://dx.doi.org/10.1139/v02-003

37. Bakleh, M. E.; Sol, V.; Granet, R.; Krausz, P.; Estieu-Gionnet, K.; Deleris, G. Tetrahedron 2009, 65, 7385-7392. http://dx.doi.org/10.1016/j.tet.2009.07.028

38. Commercially available.

39. Meier, H.; Prass, E.; Zerban, G.; Kosteyn, F. Z. Naturforsch. B 1988, 43, 889-896.

40. Meier, H.; Aust, H. J. Prakt. Chem. 1999, 341, 466-471. http://dx.doi.org/10.1002/(SICI)1521-3897(199907)341:5<466::AID-PRAC466>3.0.CO;2-V

41. Meier, H.; Lehmann, M.; Holst, H. C.; Schwöppe, D. Tetrahedron 2004, 60, 6881-6888. http://dx.doi.org/10.1016/j.tet.2004.06.012

42. Greve, S.; Näther, C.; Friedrichsen, W. Org. Lett. 2000, 2, 2269-2270. http://dx.doi.org/10.1021/o10060329, PMid:10930260

43. Lehmann, M.; Schartel, B.; Hennecke, M.; Meier, H. Tetrahedron 1999, 55, 13377-13394. http://dx.doi.org/10.1016/S0040-4020(99)00823-6

44. Figueira-Duarte, T. M.; Gegout, A.; Olivier, J.; Cardinali, F.; Nierengarten, J.-F. Eur. J. Org. Chem. 2009, 3879-3884. http://dx.doi.org/10.1002/ejoc.200900290

45. In contrast to other alkoxy substituted compounds with this stilbenoid scaffold, $18 \mathrm{a}$ and $18 \mathrm{~b}$ do not form liquid crystals. See Meier, H.; Lifka, T.; Stalmach, U.; Oehlhof, A.; Prehl, S. Eur. J. Org. Chem. 2008, 1568-1574. http://dx.doi.org/10.1002/ejoc.200701046

46. Ngwendson, J. N.; Atemnkeng, W. N.; Schultze, C. M.; Banerjee, A. Org. Lett. 2006, 8, 4085-4088.

http://dx.doi.org/10.1021/ol061594z, PMid:16928080 
47. See also Liao, L.; Pang, Y.; Ding, L.; Karasz, F. E. Macromolecules 2004, 37, 3970-3972.

48. Meier, H.; Ickenroth, D. Eur. J. Org. Chem. 2002, 1745-1749. http://dx.doi.org/10.1002/1099-0690(200206)2002:11<1745::AID-EJOC1745>3.0.CO;2-I

49. Detert, H.; Sadovski, O.; Sugiono, E. J. Phys. Org. Chem. 2004, 17, 1046-1050. http://dx.doi.org/10.1002/poc.811

50. Stalmach, U.; Kolshorn, H.; Brehm, I.; Meier, H. Liebigs Ann. Chem. 1996, 1449-1456. 